\title{
Hopf algebras and the logarithm of the S-transform in free probability
}

\author{
Mitja Mastnak Alexandru Nica 1
}

\begin{abstract}
Let $k$ be a positive integer and let $\mathcal{G}_{k}$ denote the set of all joint distributions of $k$-tuples $\left(a_{1}, \ldots, a_{k}\right)$ in a non-commutative probability space $(\mathcal{A}, \varphi)$ such that $\varphi\left(a_{1}\right)=\cdots=$ $\varphi\left(a_{k}\right)=1$. $\mathcal{G}_{k}$ is a group under the operation of free multiplicative convolution $\nabla$. We identify $\left(\mathcal{G}_{k}, \otimes\right)$ as the group of characters of a certain Hopf algebra $\mathcal{Y}^{(k)}$. Then, by using the log map from characters to infinitesimal characters of $\mathcal{Y}^{(k)}$, we introduce a transform $L S_{\mu}$ for distributions $\mu \in \mathcal{G}_{k} . L S_{\mu}$ is a power series in $k$ non-commuting indeterminates $z_{1}, \ldots, z_{k}$; its coefficients can be computed from the coefficients of the $R$-transform of $\mu$ by using summations over chains in the lattices $N C(n)$ of non-crossing partitions. The $L S$-transform has the "linearizing" property that

$$
L S_{\mu \bowtie \nu}=L S_{\mu}+L S_{\nu}, \quad \forall \mu, \nu \in \mathcal{G}_{k} \text { such that } \mu \otimes \nu=\nu \otimes \mu .
$$

In the particular case $k=1$ one has that $\mathcal{Y}^{(1)}$ is naturally isomorphic to the Hopf algebra Sym of symmetric functions, and that the $L S$-transform is very closely related to the logarithm of the $S$-transform of Voiculescu, by the formula

$$
L S_{\mu}(z)=-z \log S_{\mu}(z), \quad \forall \mu \in \mathcal{G}_{1} .
$$

In this case the group $\left(\mathcal{G}_{1}, \otimes\right)$ can be identified as the group of characters of Sym, in such a way that the $S$-transform, its reciprocal $1 / S$ and its $\operatorname{logarithm} \log S$ relate in a natural sense to the sequences of complete, elementary and respectively power sum symmetric functions.
\end{abstract}

\section{Introduction}

In this paper we study joint distributions of $k$-tuples of elements in a noncommutative probability space. Let $(\mathcal{A}, \varphi)$ be a noncommutative probability space (i.e. $\mathcal{A}$ is a unital algebra over $\mathbb{C}$ and $\varphi: \mathcal{A} \rightarrow \mathbb{C}$ is a linear functional such that $\varphi\left(1_{\mathcal{A}}\right)=1$ ), and let $a_{1}, \ldots, a_{k}$ be elements of $\mathcal{A}$. The distribution of $\left(a_{1}, \ldots, a_{k}\right)$ is the linear functional $\mu$ on the algebra of noncommutative polynomials $\mathbb{C}\left\langle X_{1}, \ldots, X_{k}\right\rangle$ defined by the requirement that

$$
\mu\left(X_{i_{1}} \cdots X_{i_{n}}\right)=\varphi\left(a_{i_{1}} \cdots a_{i_{n}}\right), \quad \forall n \geq 0, \forall 1 \leq i_{1}, \ldots, i_{n} \leq k
$$

We denote by $\mathcal{D}_{\text {alg }}(k)$ the set of linear functionals on $\mathbb{C}\left\langle X_{1}, \ldots, X_{k}\right\rangle$ that arise in this way. (Clearly, this is just the set of all linear functionals on $\mathbb{C}\left\langle X_{1}, \ldots, X_{k}\right\rangle$ such that $\mu(1)=1$.)

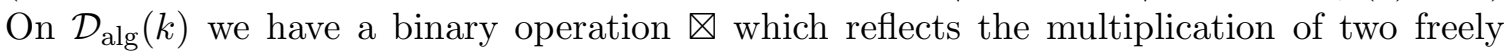
independent $k$-tuples in a noncommutative probability space. That is, $\nabla$ is well-defined and uniquely determined by the following requirement: if $a_{1}, \ldots, a_{k}, b_{1}, \ldots, b_{k}$ are elements in a noncommutative probability space $(\mathcal{A}, \varphi)$ such that $\left(a_{1}, \ldots, a_{k}\right)$ has distribution $\mu$, $\left(b_{1}, \ldots, b_{k}\right)$ has distribution $\nu$, and $\left\{a_{1}, \ldots, a_{k}\right\}$ is freely independent from $\left\{b_{1}, \ldots, b_{k}\right\}$,

\footnotetext{
${ }^{1}$ Research supported by a Discovery Grant from NSERC, Canada.
} 
then it follows that the distribution of $\left(a_{1} b_{1}, \ldots, a_{k} b_{k}\right)$ is equal to $\mu \nabla \nu$. The operation $\otimes$ on $\mathcal{D}_{\text {alg }}(k)$ is associative and unital, where the unit is the functional $\mu_{o} \in \mathcal{D}_{\text {alg }}(k)$ with $\mu_{o}\left(X_{i_{1}} \cdots X_{i_{n}}\right)=1$ for all $n \geq 1$ and $1 \leq i_{1}, \ldots, i_{n} \leq k$. A distribution $\mu \in \mathcal{D}_{\text {alg }}(k)$ is invertible with respect to $\otimes$ if and only if it satisfies $\mu\left(X_{i}\right) \neq 0, \forall 1 \leq i \leq k$; and moreover, the subset

$$
\mathcal{G}_{k}:=\left\{\mu \in \mathcal{D}_{\text {alg }}(k) \mid \mu\left(X_{i}\right)=1, \quad \forall 1 \leq i \leq k\right\}
$$

is a subgroup in the group of invertibles with respect to $\nabla$. For a basic introduction to free multiplicative convolution, we refer to Section 3.6 of [17] or to Lecture 14 in [11].

The main goal of the present paper is to introduce a transform $L S_{\mu}$ for distributions $\mu \in$ $\mathcal{G}_{k}$, which linearizes commuting $\bigotimes$-products. We arrive to the $L S$-transform by identifying $\left(\mathcal{G}_{k}, \otimes\right)$ as the group of characters of a certain Hopf algebra $\mathcal{Y}^{(k)}$. As an algebra, $\mathcal{Y}^{(k)}$ is merely a commutative algebra of polynomials:

$$
\mathcal{Y}^{(k)}=\mathbb{C}\left[Y_{w}\left|w \in[k]^{*},\right| w \mid \geq 2\right]
$$

where $[k]^{*}$ is the set of all words of finite length made with letters from the alphabet $\{1, \ldots, k\}$, and where $|w|$ denotes the length (i.e. number of letters) of a word $w \in[k]^{*}$. The feature which relates $\mathcal{Y}^{(k)}$ to free probability is its comultiplication: for $w \in[k]^{*}$ with $|w|=n \geq 2$, the comultiplication $\Delta\left(Y_{w}\right) \in \mathcal{Y}^{(k)} \otimes \mathcal{Y}^{(k)}$ is defined by using a special type of summation over the lattice of non-crossing partitions $N C(n)$ which is known (see e.g. Lectures 14 and 17 of [11]) to relate to the multiplication of free random variables. The details of the construction of $\mathcal{Y}^{(k)}$ are presented in Section 3 below. Here we only mention the fact that $\mathcal{Y}^{(k)}$ is a graded connected Hopf algebra, where every generator $Y_{w}$ from (1.2) is homogeneous of degree $|w|-1$. A consequence of this fact which is important for the present paper is that one can then define the exponential $\exp \xi$ for every linear functional $\xi: \mathcal{Y}^{(k)} \rightarrow \mathbb{C}$ such that $\xi(1)=0$, and one can define the logarithm $\log \eta$ for every linear functional $\eta: \mathcal{Y}^{(k)} \rightarrow \mathbb{C}$ such that $\eta(1)=1$. Moreover, the maps exp and log are bijections inverse to each other, between the two sets of linear functionals mentioned above.

Let $\mathbb{X}\left(\mathcal{Y}^{(k)}\right)$ be the set of characters of $\mathcal{Y}^{(k)}$,

$$
\mathbb{X}\left(\mathcal{Y}^{(k)}\right):=\left\{\eta: \mathcal{Y}^{(k)} \rightarrow \mathbb{C} \mid \eta(1)=1, \eta \text { is linear and multiplicative }\right\} .
$$

Then the logarithm map sends $\mathbb{X}\left(\mathcal{Y}^{(k)}\right)$ bijectively onto the set $\mathbb{I}\left(\mathcal{Y}^{(k)}\right)$ of infinitesimal characters of $\mathcal{Y}^{(k)}$; the latter set is defined by

$$
\mathbb{I}\left(\mathcal{Y}^{(k)}\right):=\left\{\begin{array}{l|l}
\xi: \mathcal{Y}^{(k)} \rightarrow \mathbb{C} & \begin{array}{l}
\xi \text { is linear and satisfies } \\
\xi(P Q)=\xi(P) \varepsilon(Q)+\varepsilon(P) \xi(Q), \quad \forall P, Q \in \mathcal{Y}^{(k)}
\end{array}
\end{array}\right\},
$$

where $\varepsilon$ is the counit of $\mathcal{Y}^{(k)}\left(\varepsilon \in \mathbb{X}\left(\mathcal{Y}^{(k)}\right)\right.$, and is uniquely determined by the requirement that $\varepsilon\left(Y_{w}\right)=0$ for every $w \in[k]^{*}$ with $\left.|w| \geq 2\right)$. Let us also record the facts that $\mathbb{X}\left(\mathcal{Y}^{(k)}\right)$ is a group under the operation of convolution for linear functionals on $\mathcal{Y}^{(k)}$, and that the $\log$ map linearizes commuting convolution products:

$$
\left(\eta_{1}, \eta_{2} \in \mathbb{X}\left(\mathcal{Y}^{(k)}\right), \eta_{1} \eta_{2}=\eta_{2} \eta_{1}\right) \Rightarrow \log \left(\eta_{1} \eta_{2}\right)=\log \eta_{1}+\log \eta_{2}
$$

(In this paper the convolution of linear functionals on a Hopf algebra is denoted as a plain multiplication. Our conventions of notation and a few background facts about graded connected Hopf algebras are collected in Section 2C of the paper.) 
So now let us state precisely what is the connection between the Hopf algebra $\mathcal{Y}^{(k)}$ and the operation $\otimes$. Let $\mu$ be a distribution in $\mathcal{G}_{k}$, and let us consider its $R$-transform $R_{\mu}$; this is a power series in $k$ non-commuting indeterminates $z_{1}, \ldots, z_{k}$, of the form

$$
R_{\mu}\left(z_{1}, \ldots, z_{k}\right)=\sum_{i=1}^{k} z_{i}+\sum_{\substack{w \in[k]^{*},|w| \geq 2}} \alpha_{w} z_{w},
$$

where in the latter sum we used the shorthand notation $z_{w}:=z_{i_{1}} \cdots z_{i_{n}}$ for $w=\left(i_{1}, \ldots, i_{n}\right) \in$ $[k]^{*}$ with $n \geq 2$. A brief review of how the coefficients $\alpha_{w}$ are calculated from the moments (i.e. values on monomials) of $\mu$ is made in Section 2B below - see Definition 2.5, Remark 2.6 ,

Definition 1.1. Let $\mu$ be a distribution in $\mathcal{G}_{k}$ and consider the $R$-transform $R_{\mu}$, denoted as in Equation (1.6). The character of $\mathcal{Y}^{(k)}$ associated to $\mu$ is the character $\chi_{\mu} \in \mathbb{X}\left(\mathcal{Y}^{(k)}\right)$ uniquely determined by the requirement that

$$
\chi_{\mu}\left(Y_{w}\right)=\alpha_{w}, \quad \forall w \in[k]^{*} \text { such that }|w| \geq 2 .
$$

Theorem 1.2. The map $\mu \mapsto \chi_{\mu}$ defined above is a group isomorphism from $\left(\mathcal{G}_{k}, \nabla\right)$ onto the group $\mathbb{X}\left(\mathcal{Y}^{(k)}\right)$ of characters of $\mathcal{Y}^{(k)}$ (endowed with the operation of convolution).

The proof of Theorem 1.2 is presented in Section 3 below. Let us mention that the proof is quite short, and follows easily from known facts about how the multiplication of free $k$-tuples is described in terms of their $R$-transforms, as explained for instance in Lecture 17 of [11. (In a certain sense, we are dealing here with a situation where the convolution of characters of $\mathcal{Y}^{(k)}$ was studied before looking at $\mathcal{Y}^{(k)}$ itself.) Nevertheless, Theorem 1.2 is important because it brings to attention the fact that Hopf algebra methods can be used in the study of $\otimes$. In particular, by taking (1.5) into account, we see that Theorem 1.2 has the following immediate corollary.

Corollary 1.3. Let $\mu$ and $\nu$ be distributions in $\mathcal{G}_{k}$ such that $\mu \otimes \nu=\nu \otimes \mu$. Then

$$
\log \chi_{\mu \bowtie \nu}=\log \chi_{\mu}+\log \chi_{\nu}
$$

where the characters $\chi_{\mu}, \chi_{\nu}$ and $\chi_{\mu \rrbracket_{\nu}}$ are as in Definition 1.1, and their logarithms are the corresponding functionals from $\mathbb{I}\left(\mathcal{Y}^{(k)}\right)$.

The $L S$-transform $L S_{\mu}$ is defined so that it stores the information about the infinitesimal character $\log \chi_{\mu}$, as follows.

Definition 1.4. Let $\mu$ be a distribution in $\mathcal{G}_{k}$. The $L S$-transform of $\mu$ is the power series

$$
L S_{\mu}\left(z_{1}, \ldots, z_{k}\right):=\sum_{\substack{w \in[k]^{*},|w| \geq 2}}\left(\left(\log \chi_{\mu}\right)\left(Y_{w}\right)\right) z_{w}
$$

where $\log \chi_{\mu} \in \mathbb{I}\left(\mathcal{Y}^{(k)}\right)$ is as in Corollary 1.3, and where the meaning of " $z_{w}$ " is same as in Equation (1.6).

Clearly, Corollary 1.3 can also be phrased as a statement about $L S$-transforms. In this guise, it simply says that the $L S$-transform linearizes commuting $\bigotimes$-products: 
Corollary 1.5. Let $\mu$ and $\nu$ be distributions in $\mathcal{G}_{k}$ such that $\mu \nabla \nu=\nu \otimes \mu$. Then

$$
L S_{\mu \bowtie \nu}=L S_{\mu}+L S_{\nu}
$$

In particular, formula (1.10) always applies when one of $\mu, \nu$ is the joint distribution of a repeated $k$-tuple $(a, a, \ldots, a)$, where $a$ is a random variable in a non-commutative probability space $(\mathcal{A}, \varphi)$. A version of this fact which lives in the framework of a $C^{*}$-probability space is discussed in Example 5.2 below.

The $L S$-transform was introduced above by using the Hopf algebra $\mathcal{Y}^{(k)}$, but it can also be described directly in combinatorial terms, by using summations over chains in lattices of non-crossing partitions. We next explain how this goes.

A chain in $N C(n)$ is an object of the form

$$
\Gamma=\left(\pi_{0}, \pi_{1}, \ldots, \pi_{\ell}\right)
$$

with $\pi_{0}, \pi_{1}, \ldots, \pi_{\ell} \in N C(n)$ such that $0_{n}=\pi_{0}<\pi_{1}<\cdots<\pi_{\ell}=1_{n}$ (and where $0_{n}$ and $1_{n}$ denote the minimal and maximal element of $N C(n)$, respectively). For a chain $\Gamma$ as in (1.11), the number $\ell$ is called the length of $\Gamma$ and will be denoted as $|\Gamma|$. Given a formal power series $f$ in non-commuting indeterminates $z_{1}, \ldots, z_{k}$, one has a natural way of defining some "generalized coefficients"

$$
\mathrm{Cf}_{\left(i_{1}, \ldots, i_{n}\right)}^{(\Gamma)}(f)
$$

where $n \geq 2,1 \leq i_{1}, \ldots, i_{n} \leq k$, and $\Gamma$ is a chain in $N C(n)$. Every generalized coefficient (1.12) is defined to be a certain product of actual coefficients of $f$ (see Definition 4.3 below for the precise formula). By using the generalized coefficients (1.12), the combinatorial description of the $L S$-transform is stated as follows.

Theorem 1.6. Let $\mu$ be a distribution in $\mathcal{G}_{k}$, and let $w=\left(i_{1}, \ldots, i_{n}\right)$ be a word in $[k]^{*}$, where $n \geq 2$. Consider (as in Equation (1.9) of Definition 1.4) the coefficient $\left(\log \chi_{\mu}\right)\left(Y_{w}\right)$ of $z_{w}$ in the LS-transform of $\mu$. This coefficient can be also expressed as

$$
\left(\log \chi_{\mu}\right)\left(Y_{w}\right)=\sum_{\substack{\Gamma \text { chain } \\ \text { in } N C(n)}} \frac{(-1)^{1+|\Gamma|}}{|\Gamma|} C f_{\left(i_{1}, \ldots, i_{n}\right)}^{(\Gamma)}\left(R_{\mu}\right),
$$

where $R_{\mu}$ is the $R$-transform of $\mu$.

Remark 1.7. $1^{o} N C(n)$ has a unique chain of length 1 (namely the chain $\left(0_{n}, 1_{n}\right)$ ); the term indexed by this chain in the sum on the right-hand side of (1.13) is precisely the coefficient of $z_{w}$ in $R_{\mu}$, while every other term of the same sum turns out to be a product of coefficients of $R_{\mu}$ with lengths strictly smaller than $n$. From this observation it is immediate that the coefficients of $R_{\mu}$ can be computed back, recursively, in terms of the coefficients of $L S_{\mu}$. Moreover, since $\mu$ is completely determined by its $R$-transform, we thus see that $\mu$ is completely determined by the series $L S_{\mu}$ as well.

$2^{o}$ By using Theorem [1.6 one finds that the $L S$-transform inherits a fundamental property that the $R$-transform is known to have in connection to free independence: a $k$-tuple in a noncommutative probability space is freely independent if and only if the $L S$-transform of its distribution separates the variables. The precise statement of this fact appears as Proposition 5.4 below. 
In the remaining part of the introduction we look at the particular case when $k=1$. In this case the notations are simplified due to the fact that words over the 1-letter alphabet $\{1\}$ are determined by their lengths. We make the convention to write simply " $Y_{n}$ " instead of $Y_{(1,1, \ldots, 1)}$ with $n$ repetitions of 1 in the index; thus Equation (1.2) is now written in the form

$$
\mathcal{Y}^{(1)}=\mathbb{C}\left[Y_{n} \mid n \geq 2\right],
$$

while Equation (1.9) defining $L S_{\mu}$ reduces to

$$
L S_{\mu}(z)=\sum_{n=2}^{\infty}\left(\left(\log \chi_{\mu}\right)\left(Y_{n}\right)\right) z^{n} .
$$

A special feature of the case $k=1$ (not holding for $k \geq 2$ ) is that the operation $\otimes$ is commutative. Hence for $k=1$ the linearization property stated in Corollary [1.5] holds for all $\mu, \nu \in \mathcal{G}_{1}$.

Now, in the case $k=1$ there exists an established way of treating multiplicative free convolution, by using Voiculescu's $S$-transform. The $S$-transform $S_{\mu}$ of a distribution $\mu \in \mathcal{G}_{1}$ is a power series in an indeterminate $z$, with constant term equal to 1 . Taking $S$-transforms converts $\otimes$ into plain multiplication of power series:

$$
S_{\mu 凶 \nu}(z)=S_{\mu}(z) \cdot S_{\nu}(z), \quad \forall \mu, \nu \in \mathcal{G}_{1}
$$

(see Section 3.6 in [17], or Lecture 18 in [1]). We prove that the one-dimensional $L S$ transform is related to the $S$-transform, as follows.

Theorem 1.8. For a distribution $\mu \in \mathcal{G}_{1}$, the power series $S_{\mu}$ and $L S_{\mu}$ are related by

$$
L S_{\mu}(z)=-z \log S_{\mu}(z) .
$$

Theorem 1.8 shows that the multiplicativity of the $S$-transform can be retrieved from the particular case $k=1$ of Corollary 1.5, one only needs to divide by $-z$ and then exponentiate both sides of Equation (1.10). We should note here that this exponentiating trick is specific to the 1-variable situation, for $k \geq 2$ it is probably better to work with $L S_{\mu}$ itself rather than considering its exponential. (Indeed, for $k \geq 2$ it isn't generally true that the $k$-variable series $L S_{\mu}$ and $L S_{\nu}$ would commute, even if $\mu, \nu \in \mathcal{G}_{k}$ are such that $\mu \otimes \nu=\nu \otimes \mu$. Hence when one exponentiates Equation (1.10) for $k \geq 2$, the series appearing on the right-hand side isn't generally equal to $\exp \left(L S_{\mu}\right) \cdot \exp \left(L S_{\nu}\right)$.)

The proof of Theorem 1.8 is obtained by following the connections that $\mathcal{Y}^{(1)}$ has with symmetric functions. We start from the fact that (independently of the isomorphism $\mathcal{G}_{1} \ni$ $\mu \mapsto \chi_{\mu} \in \mathbb{X}\left(\mathcal{Y}^{(1)}\right)$ from Theorem 1.2) one can also find a natural group isomorphism $\mu \mapsto \theta_{\mu}$ from $\left(\mathcal{G}_{1}, \bigotimes\right)$ onto the group of characters of the Hopf algebra Sym of symmetric functions. The map $\mu \mapsto \theta_{\mu}$ is defined in such a way that the $S$-transform, its reciprocal $1 / S$ and its $\operatorname{logarithm} \log S$ relate in a natural sense to the sequences of complete, elementary and respectively power sum symmetric functions (see Remark 6.7 below for the precise description of how this happens). We then concretely put into evidence an isomorphism $\Phi: \mathcal{Y}^{(1)} \rightarrow$ Sym which has the property that

$$
\chi_{\mu}=\theta_{\mu} \circ \Phi, \quad \forall \mu \in \mathcal{G}_{1}
$$

with the help of $\Phi$ we can place the 1-dimensional $L S$-transform in the framework of Sym, and then prove the relation stated in Theorem 1.8 (this is done in Proposition 6.10 and Corollary 6.12). 
It is interesting to note that the way the $L S$-transform is introduced in this paper is close in spirit to how the $S$-transform was first found by Voiculescu in [16]. Some other approaches to the $S$-transform were found in the meanwhile (e.g. in [6] or in [10]), but the one from [16] has the distinctive feature that it relies on the exponential map for a certain commutative Lie group, constructed to reflect how the moments of $\mu \otimes \nu$ are computed in terms of the moments of $\mu$ and of $\nu\left(\mu, \nu \in \mathcal{G}_{1}\right)$. The approach in the present paper goes on the same lines, with the difference that it insists on structures (free cumulants, Hopf algebras) where it is easier to pursue a detailed combinatorial analysis. The possibility of moving up to $L S$-transforms for $k$-tuples then comes as a manifestation of the general principle that combinatorial arguments in free probability often extend without much trouble from the 1 -variable to the $k$-variable setting.

We conclude this introduction by describing how the paper is organized. Besides the introduction, the paper has six other sections. Section 2 contains a review of some background and notations. In Section 3 we introduce the Hopf algebra $\mathcal{Y}^{(k)}$ and we prove Theorem 1.2, then Section 4 is devoted to the combinatorics of the $L S$-transform and to the proof of Theorem 1.6. In Section 5 we discuss some basic properties of $L S_{\mu}$. The last two sections of the paper are devoted to the one-variable framework: in Section 6 we prove Theorem 1.8 and in Section 7 we discuss in more detail the isomorphism between $\mathcal{Y}^{(1)}$ and the Hopf algebra Sym of symmetric functions.

\section{Background and notations}

\section{A. Non-crossing partitions}

Notation 2.1. $1^{o}$ We will use the standard conventions of notation for non-crossing partitions (as in [13], or in Lecture 9 of [11]). For a positive integer $n$, the set of all non-crossing partitions of $\{1, \ldots, n\}$ will be denoted by $N C(n)$. For $\pi \in N C(n)$, the number of blocks of $\pi$ will be denoted by $|\pi|$. On $N C(n)$ we consider the partial order given by reversed refinement: for $\pi, \rho \in N C(n)$, we write " $\pi \leq \rho$ " to mean that every block of $\rho$ is a union of blocks of $\pi$. The minimal and maximal element of $(N C(n), \leq)$ are denoted by $0_{n}$ (the partition of $\{1, \ldots, n\}$ into $n$ blocks of 1 element each) and respectively $1_{n}$ (the partition of $\{1, \ldots, n\}$ into 1 block of $n$ elements).

$2^{o}$ Every partition $\pi \in N C(n)$ has associated to it a permutation of $\{1, \ldots, n\}$, which is denoted by $P_{\pi}$, and is defined by the following prescription: for every block $B=\left\{b_{1}, \ldots, b_{m}\right\}$ of $\pi$, with $b_{1}<\cdots<b_{m}$, one creates a cycle of $P_{\pi}$ by putting

$$
P_{\pi}\left(b_{1}\right)=b_{2}, \ldots, P_{\pi}\left(b_{m-1}\right)=b_{m}, P_{\pi}\left(b_{m}\right)=b_{1} .
$$

Note that in the particular case when $\pi=0_{n}$ we have that $P_{0_{n}}$ is the identical permutation of $\{1, \ldots, n\}$, while for $\pi=1_{n}$ we have that $P_{1_{n}}$ is the cycle $1 \mapsto 2 \mapsto \cdots \mapsto n \mapsto 1$.

$3^{o}$ The Kreweras complementation map is a special order-reversing bijection $K: N C(n) \rightarrow$ $N C(n)$. In this paper we will use its description in terms of permutations associated to non-crossing partitions: for $\pi \in N C(n)$, the Kreweras complement of $\pi$ is the partition $K(\pi) \in N C(n)$ uniquely determined by the fact that its associated permutation is

$$
P_{K(\pi)}=P_{\pi}^{-1} P_{1_{n}}
$$


Formula (2.1) can be extended in order to cover the concept of relative Kreweras complement of $\pi$ in $\rho$, for $\pi, \rho \in N C(n)$ such that $\pi \leq \rho$. This is the partition in $N C(n)$, denoted by $K_{\rho}(\pi)$, uniquely determined by the fact that the permutation associated to it is

$$
P_{K_{\rho}(\pi)}=P_{\pi}^{-1} P_{\rho}
$$

Clearly, the Kreweras complementation map $K$ from (2.1) is the relative complementation with respect to the maximal element $1_{n}$ of $N C(n)$.

The formulas (2.1), (2.2) do not follow exactly the original approach used by Kreweras in [8], but are easily seen to be equivalent to it (see e.g. [11], Exercise 18.25 on p. 301).

Remark 2.2. In this remark we record a few facts about relative Kreweras complements that will be used later on in the paper.

$1^{o}$ For $\pi \leq \rho$ in $N C(n)$, the number of blocks of the relative Kreweras complement $K_{\rho}(\pi)$ is

$$
\left|K_{\rho}(\pi)\right|=n+|\rho|-|\pi|
$$

(see e.g. [11, Exercise 18.23 on p. 300).

$2^{o}$ For a fixed partition $\rho \in N C(n)$, the relative Kreweras complements $K_{\rho}(\pi)$ of partitions $\pi \leq \rho$ can be obtained by taking "separate Kreweras complements" inside each block of $\rho$. More precisely, let us write explicitly $\rho=\left\{B_{1}, \ldots, B_{q}\right\}$. It is easy to see that one has a natural poset isomorphism

$$
\{\pi \in N C(n) \mid \pi \leq \rho\} \ni \pi \mapsto\left(\pi_{1}, \ldots, \pi_{q}\right) \in N C\left(\left|B_{1}\right|\right) \times \cdots \times N C\left(\left|B_{q}\right|\right)
$$

where for every $1 \leq j \leq q$ the partition $\pi_{j} \in N C\left(\left|B_{j}\right|\right)$ is obtained by restricting $\pi$ to $B_{j}$ and by re-denoting the elements of $B_{j}$, in increasing order, so that they become $1,2, \ldots,\left|B_{j}\right|$. The above statement about taking separate complements inside each block of $\rho$ then amounts to the fact that for $\pi \leq \rho$ in $N C(n)$ we have the implication

$$
\left(\pi \mapsto\left(\pi_{1}, \ldots, \pi_{q}\right)\right) \Rightarrow\left(K_{\rho}(\pi) \mapsto\left(K\left(\pi_{1}\right), \ldots, K\left(\pi_{q}\right)\right)\right),
$$

where $K\left(\pi_{1}\right), \ldots, K\left(\pi_{q}\right)$ are Kreweras complements calculated in $N C\left(\left|B_{1}\right|\right), \ldots, N C\left(\left|B_{q}\right|\right)$, respectively. For a discussion of this, see pp. 288-290 in Lecture 18 of [11].

$3^{o}$ From part $2^{o}$ of this remark it is immediate that, for a fixed $\rho \in N C(n)$, the map $\pi \mapsto K_{\rho}(\pi)$ is an order-reversing bijection from $\{\pi \in N C(n) \mid \pi \leq \rho\}$ onto itself. On the other hand, it can be shown that

$$
\pi \leq \rho_{1} \leq \rho_{2} \text { in } N C(n) \Rightarrow K_{\rho_{1}}(\pi) \leq K_{\rho_{2}}(\pi)
$$

(see Exercise 18.25.1 on p. 301 of [11]). Hence the expression " $K_{\rho}(\pi)$ " is increasing as a function of $\rho$, and is decreasing as a function of $\pi$.

The particular case when $\rho_{2}=1_{n}$ in (2.6) gives us that

$$
\pi \leq \rho \text { in } N C(n) \Rightarrow K_{\rho}(\pi) \leq K(\pi) .
$$

Thus for every $\pi \in N C(n)$ it makes sense to define a map

$$
\left\{\begin{aligned}
\{\rho \in N C(n) \mid \rho \geq \pi\} & \rightarrow\{\sigma \in N C(n) \mid \sigma \leq K(\pi)\} \\
\rho & \mapsto K_{\rho}(\pi) .
\end{aligned}\right.
$$


This map turns out to be bijective, and a helpful fact for calculating its inverse is that

$$
\left(\rho \geq \pi, K_{\rho}(\pi)=\sigma\right) \Rightarrow K_{K(\pi)}(\sigma)=K(\rho)
$$

(see Lemma 18.9 and Remark 18.10 on p. 291 of [11]). Finally, let us also record here the fact that the whole family of bijections in (2.8) (corresponding to the various partitions $\pi \in N C(n))$ can be consolidated into one bijection,

$$
\left\{\begin{aligned}
\{(\pi, \rho) \mid \pi, \rho \in N C(n), \pi \leq \rho\} & \rightarrow\{(\pi, \sigma) \mid \pi, \sigma \in N C(n), \sigma \leq K(\pi)\} \\
(\pi, \rho) & \mapsto\left(\pi, K_{\rho}(\pi)\right)
\end{aligned}\right.
$$

\section{B. Power series}

Here we review a few relevant notations and facts about series in non-commuting indeterminates, and in particular about multivariable $R$-transforms.

Notation 2.3. Let $k$ be a positive integer. As already mentioned in the introduction, we use the notation $[k]^{*}$ for the set of all words of finite length over the alphabet $\{1, \ldots, k\}$ :

$$
[k]^{*}:=\cup_{n=0}^{\infty}\{1, \ldots, k\}^{n} .
$$

The length of a word $w \in[k]^{*}$ will be denoted by $|w|$. (In (2.11) we followed the standard procedure of also including into $[k]^{*}$ a unique word $\phi$ with $|\phi|=0$.)

Definition 2.4. (Series and their coefficients.) Let $k$ be a positive integer.

$1^{o}$ We will use the notation $\mathbb{C}_{0}\left\langle\left\langle z_{1}, \ldots, z_{k}\right\rangle\right\rangle$ for the set of power series with complex coefficients and with vanishing constant term in the non-commuting indeterminates $z_{1}, \ldots, z_{k}$. The general form of a series $f \in \mathbb{C}_{0}\left\langle\left\langle z_{1}, \ldots, z_{k}\right\rangle\right\rangle$ is thus

$$
f\left(z_{1}, \ldots, z_{k}\right)=\sum_{n=1}^{\infty} \sum_{i_{1}, \ldots, i_{n}=1}^{k} \alpha_{\left(i_{1}, \ldots, i_{n}\right)} z_{i_{1}} \cdots z_{i_{n}}=\sum_{\substack{w \in[k]^{*},|w| \geq 1}} \alpha_{w} z_{w}
$$

where the coefficients $\alpha_{w}$ are from $\mathbb{C}$ and where, same as in the introduction, we write in short $z_{w}:=z_{i_{1}} \cdots z_{i_{n}}$ for $w=\left(i_{1}, \ldots, i_{n}\right) \in\{1, \ldots, k\}^{n}, n \geq 1$.

$2^{o}$ For every word $w \in[k]^{*}$ with $|w| \geq 1$ we will denote by

$$
\mathrm{Cf}_{w}: \mathbb{C}_{0}\left\langle\left\langle z_{1}, \ldots, z_{k}\right\rangle\right\rangle \rightarrow \mathbb{C}
$$

the linear functional which extracts the coefficient of $z_{w}$ in a series $f \in \mathbb{C}_{0}\left\langle\left\langle z_{1}, \ldots, z_{k}\right\rangle\right\rangle$. Thus for $f$ written as in Equation (2.12) we have $\mathrm{Cf}_{w}(f)=\alpha_{w}$.

$3^{o}$ Suppose we are given a positive integer $n$, a word $w=\left(i_{1}, \ldots, i_{n}\right) \in\{1, \ldots, k\}^{n}$ and a partition $\pi \in N C(n)$. We define a (generally non-linear) functional

$$
\mathrm{Cf}_{w ; \pi}: \mathbb{C}_{0}\left\langle\left\langle z_{1}, \ldots, z_{k}\right\rangle\right\rangle \rightarrow \mathbb{C}
$$

as follows. For every block $B=\left\{b_{1}, \ldots, b_{m}\right\}$ of $\pi$, with $1 \leq b_{1}<\cdots<b_{m} \leq n$, let us use the notation

$$
w\left|B=\left(i_{1}, \ldots, i_{n}\right)\right| B:=\left(i_{b_{1}}, \ldots, i_{b_{m}}\right) \in\{1, \ldots, k\}^{m} .
$$


Then we define

$$
\mathrm{Cf}_{w ; \pi}(f):=\prod_{B \text { block of } \pi} \mathrm{Cf}_{w \mid B}(f), \quad \forall f \in \mathbb{C}_{0}\left\langle\left\langle z_{1}, \ldots, z_{k}\right\rangle\right\rangle .
$$

(For example if $w=\left(i_{1}, \ldots, i_{5}\right)$ is a word of length 5 and if $\pi=\{\{1,4,5\},\{2,3\}\} \in$ $N C(5)$, then the above formula comes to $\mathrm{Cf}_{\left(i_{1}, i_{2}, i_{3}, i_{4}, i_{5}\right) ; \pi}(f)=\mathrm{Cf}_{\left(i_{1}, i_{4}, i_{5}\right)}(f) \cdot \mathrm{Cf}_{\left(i_{2}, i_{3}\right)}(f)$, $f \in \mathbb{C}_{0}\left\langle\left\langle z_{1}, \ldots, z_{k}\right\rangle\right\rangle$.)

Definition 2.5. Let $\mu$ be a distribution in $\mathcal{D}_{\text {alg }}(k)$ (that is, $\mu: \mathbb{C}\left\langle X_{1}, \ldots, X_{k}\right\rangle \rightarrow \mathbb{C}$ is a linear functional such that $\mu(1)=1)$. The $R$-transform of $\mu$ is the series $R_{\mu} \in \mathbb{C}_{0}\left\langle\left\langle z_{1}, \ldots, z_{k}\right\rangle\right\rangle$ uniquely determined by the requirement that for every $n \geq 1$ and every $1 \leq i_{1}, \ldots, i_{n} \leq k$ one has

$$
\mu\left(X_{i_{1}} \cdots X_{i_{n}}\right)=\sum_{\pi \in N C(n)} \mathrm{Cf}_{\left(i_{1}, \ldots, i_{n}\right) ; \pi}\left(R_{\mu}\right) .
$$

Remark 2.6. It is easy to see that Equation (2.15) does indeed determine a unique series in $\mathbb{C}_{0}\left\langle\left\langle z_{1}, \ldots, z_{k}\right\rangle\right\rangle$. The coefficients of $R_{\mu}$ are called the free cumulants of $\mu$, and because of this reason Equation (2.15) is sometimes referred to as the (free) "moment-cumulant formula" - see Lectures 11 and 16 in [11.

Observe that, for $n=1$, Equation (2.15) simply says that $\mathrm{Cf}_{(i)}\left(R_{\mu}\right)=\mu\left(X_{i}\right), 1 \leq i \leq k$. This explains why for $\mu \in \mathcal{G}_{k}$ the $R$-transform $R_{\mu}$ was displayed in Equation (1.6) of the introduction by having all its linear coefficients equal to 1 .

An important point for the present paper is that the $R$-transform has a very nice behaviour under the operation $\otimes$. This is recorded in the next proposition.

Proposition 2.7. Let $\mu, \nu$ be distributions in $\mathcal{D}_{\text {alg }}(k)$, and let $w$ be a word in $[k]^{*}$, with $|w| \geq 1$. Then

$$
C f_{w}\left(R_{\mu \otimes \nu}\right)=\sum_{\pi \in N C(n)} C f_{w ; \pi}\left(R_{\mu}\right) \cdot C f_{w ; K(\pi)}\left(R_{\nu}\right) .
$$

For the proof of Proposition 2.7 we refer to Theorem 14.4 and Proposition 17.2 of [1].

\section{C. Graded connected Hopf algebras}

We will work with graded bialgebras over $\mathbb{C}$ and we will use the standard conventions for notations regarding them (as in the monograph [15], for instance). Our review here does not aim at generality, but just covers the specialized Hopf algebras used in the present paper.

Notation 2.8. Let $\mathcal{B}$ be a graded bialgebra over $\mathbb{C}$.

$1^{o}$ The comultiplication and counit of $\mathcal{B}$ will be denoted by $\Delta$ and respectively $\varepsilon$ (or by $\Delta_{\mathcal{B}}$ and $\varepsilon_{\mathcal{B}}$ when necessary to distinguish $\mathcal{B}$ from other graded bialgebras that are considered at the same time). The iterations of $\Delta$ will be denoted as $\Delta^{\ell}, \ell \geq 1$. Thus every $\Delta^{\ell}$ is a linear map from $\mathcal{B}$ to $\mathcal{B}^{\otimes \ell}$, where $\Delta^{1}=$ id (the identity map from $\mathcal{B}$ to $\mathcal{B}$ ), $\Delta^{2}=\Delta$, and for $\ell \geq 3$ we put

$$
\Delta^{\ell}:=(\Delta \otimes \underbrace{\mathrm{id} \otimes \cdots \otimes \mathrm{id}}_{\ell-2}) \circ \Delta^{\ell-1}
$$


$2^{o}$ For every $n \geq 0$, the vector subspace of $\mathcal{B}$ which consists of homogeneous elements of degree $n$ will be denoted by $\mathcal{B}_{n}$. We thus have a direct sum decomposition $\mathcal{B}=\oplus_{n=0}^{\infty} \mathcal{B}_{n}$ where

$$
\left\{\begin{array} { l } 
{ \mathcal { B } _ { 0 } \ni 1 _ { \mathcal { B } } ( \text { the unit of } \mathcal { B } ) , } \\
{ \mathcal { B } _ { m } \cdot \mathcal { B } _ { n } \subseteq \mathcal { B } _ { m + n } , \quad \forall m , n \geq 0 , }
\end{array} \quad \text { and } \quad \left\{\begin{array}{l}
\left.\varepsilon\right|_{\mathcal{B}_{n}}=0, \quad \forall n \geq 1 \\
\Delta\left(\mathcal{B}_{n}\right) \subseteq \oplus_{i=0}^{n} \mathcal{B}_{i} \otimes \mathcal{B}_{n-i}, \quad \forall n \geq 0
\end{array}\right.\right.
$$

If the space $\mathcal{B}_{0}$ of homogeneous elements of degree 0 is equal to $\mathbb{C} 1_{\mathcal{B}}$ then we say that the graded bialgebra $\mathcal{B}$ is connected.

Remark 2.9. (The convolution algebra $L(\mathcal{B}, \mathcal{M})$.) Let $\mathcal{B}$ be a graded connected bialgebra, let $\mathcal{M}$ be a unital algebra over $\mathbb{C}$, and let $L(\mathcal{B}, \mathcal{M})$ denote the vector space of all linear maps from $\mathcal{B}$ to $\mathcal{M}$. For $\xi, \eta \in L(\mathcal{B}, \mathcal{M})$ one can define their convolution product, denoted here simply as " $\xi \eta$ ", by the formula

$$
\xi \eta:=\text { Mult } \circ(\xi \otimes \eta) \circ \Delta,
$$

where Mult $: \mathcal{M} \otimes \mathcal{M} \rightarrow \mathcal{M}$ is the linear map given by multiplication (Mult $(x \otimes y)=x y$ for $x, y \in \mathcal{M})$. In other words, (2.18) says that for $b \in \mathcal{B}$ with $\Delta(b)=\sum_{i=1}^{n} b_{i}^{\prime} \otimes b_{i}^{\prime \prime}$ one has

$$
(\xi \eta)(b):=\sum_{i=1}^{n} \xi\left(b_{i}^{\prime}\right) \eta\left(b_{i}^{\prime \prime}\right) \in \mathcal{M} .
$$

When endowed with its usual vector space structure and with the convolution product, $L(\mathcal{B}, \mathcal{M})$ becomes itself a unital algebra over $\mathbb{C}$. The unit of $L(\mathcal{B}, \mathcal{M})$ is the linear map $\mathcal{B} \ni b \mapsto \varepsilon(b) 1_{\mathcal{M}}$, which by a slight abuse of notation is still denoted as $\varepsilon$ (same notation as for the counit of $\mathcal{B})$.

Let us also record here that when one considers a convolution product of $\ell \geq 1$ elements $\xi_{1}, \ldots, \xi_{\ell} \in L(\mathcal{B}, \mathcal{M})$, then Equation (2.18) becomes

$$
\xi_{1} \xi_{2} \cdots \xi_{\ell}=\operatorname{Mult}_{\ell} \circ\left(\xi_{1} \otimes \cdots \otimes \xi_{\ell}\right) \circ \Delta^{\ell},
$$

where $\Delta^{\ell}: \mathcal{B} \rightarrow \mathcal{B}^{\otimes \ell}$ is the $\ell$ th iteration of the comultiplication and Mult $:_{\ell} \mathcal{M}^{\otimes \ell} \rightarrow \mathcal{M}$ is given by multiplication.

For every $\xi \in L(\mathcal{B}, \mathcal{M})$ it makes sense to form polynomial expressions in $\xi$, that is, expressions of the form

$$
\sum_{\ell=0}^{n} t_{\ell} \xi^{\ell} \in L(\mathcal{B}, \mathcal{M}), \quad \text { for } n \geq 0 \text { and } t_{0}, t_{1}, \ldots, t_{n} \in \mathbb{C},
$$

where the $\xi^{\ell}(0 \leq \ell \leq n)$ are convolution powers of $\xi$, and we make the convention that $\xi^{0}:=\varepsilon$ (the unit of $L(\mathcal{B}, \mathcal{M})$ ). An important point for the present paper is that if $\xi$ is such that $\xi\left(1_{\mathcal{B}}\right)=0$ then it also makes sense to define an element

$$
\eta:=\sum_{\ell=0}^{\infty} t_{\ell} \xi^{\ell} \in L(\mathcal{B}, \mathcal{M})
$$

for an arbitrary infinite sequence $\left(t_{\ell}\right)_{\ell \geq 0}$ in $\mathbb{C}$. Indeed, if $\xi\left(1_{\mathcal{B}}\right)=0$ then by using (2.20) and the fact that $\Delta$ respects the grading one immediately sees that $\xi^{\ell}$ vanishes on $\mathcal{B}_{n}$ whenever $\ell>n$. Thus $\eta$ from (2.21) can be defined as the unique linear map from $\mathcal{B}$ to $\mathcal{M}$ which satisfies

$$
\left.\eta\right|_{\mathcal{B}_{n}}=\left.\left(\sum_{\ell=0}^{N} t_{\ell} \xi^{\ell}\right)\right|_{\mathcal{B}_{n}}, \quad \forall N \geq n \geq 0 .
$$


Remark 2.10. (The antipode.) Here we consider the special case of Remark 2.9 where $\mathcal{M}=\mathcal{B}$, and we observe that $\mathcal{B}$ is sure to be a Hopf algebra - this means, by definition, that the identity map id $: \mathcal{B} \rightarrow \mathcal{B}$ is an invertible element in the convolution algebra $L(\mathcal{B}, \mathcal{B})$. The inverse of id is called the antipode of $\mathcal{B}$ and is denoted by $S$. A reason why $S$ is sure to exist is that one can introduce it via a series expansion as in (2.21) above, which mimics the geometric series expansion of $(\varepsilon-(\varepsilon-\mathrm{id}))^{-1}$. That is, one can put

$$
S:=\varepsilon+\sum_{\ell=1}^{\infty}(\varepsilon-\mathrm{id})^{\ell} \in L(\mathcal{B}, \mathcal{B})
$$

(which makes sense because $(\varepsilon-\mathrm{id})\left(1_{\mathcal{B}}\right)=0$ ), and one can then verify that $S$ from $(2.23)$ has indeed the property that $S$ id $=\varepsilon=$ id $S$. See e.g. Propositions 5.2 and 5.3 in [5], or Theorem 14 in Appendix B of [2].

A remarkable fact about $S$ (holding for the antipode of any Hopf algebra) is that it is a unital anti-homomorphism of $\mathcal{B}$, that is, one has $S\left(1_{\mathcal{B}}\right)=1_{\mathcal{B}}$ and $S\left(b b^{\prime}\right)=S\left(b^{\prime}\right) S(b), \forall b, b^{\prime} \in$ $\mathcal{B}$; see Proposition 4.0.1 in [15].

Remark 2.11. (The group of characters.) Here we consider the special case of Remark 2.9 where $\mathcal{M}=\mathbb{C}$. A unital homomorphism from $\mathcal{B}$ to $\mathbb{C}$ is called a character. The set of all characters of $\mathcal{B}$ will be denoted by $\mathbb{X}(\mathcal{B})$. It is easy to verify that the convolution product of two characters is again a character. Moreover, if $\eta$ is a character then it is obvious that the functional $\eta \circ S \in L(\mathcal{B}, \mathbb{C})$ is a character as well, and it is easy to verify that $\eta(\eta \circ S)=\varepsilon=(\eta \circ S) \eta$. Hence $\mathbb{X}(\mathcal{B})$ is a subgroup of the group of invertibles of $L(\mathcal{B}, \mathbb{C})$, and is thus referred to as the group of characters of $\mathcal{B}$.

The fact observed above, that the inverse of $\eta \in \mathbb{X}(\mathcal{B})$ is $\eta \circ S$, can be combined with Equation (2.23) from Remark 2.10 to give us a formula for calculating inverses of characters via a series expansion,

$$
\eta^{-1}=\varepsilon+\sum_{\ell=1}^{\infty}(\varepsilon-\eta)^{\ell}, \quad \text { for } \eta \in \mathbb{X}(\mathcal{B}) .
$$

Remark 2.12. (Exponentials and logarithms for functionals.) Let $\mathcal{B}$ be a graded connected Hopf algebra. If $\xi$ is a functional in $L(\mathcal{B}, \mathbb{C})$ such that $\xi\left(1_{\mathcal{B}}\right)=0$, then it makes sense to define its exponential by the familiar formula

$$
\exp \xi=\sum_{\ell=0}^{\infty} \frac{1}{\ell !} \xi^{\ell}
$$

where on the right-hand side of (2.25) we use the recipe for series reviewed in Remark 2.9], It is easy to see that exp maps bijectively the set of functionals $\left\{\xi \in L(\mathcal{B}, \mathbb{C}) \mid \xi\left(1_{\mathcal{B}}\right)=0\right\}$ onto $\left\{\eta \in L(\mathcal{B}, \mathbb{C}) \mid \eta\left(1_{\mathcal{B}}\right)=1\right\}$; the inverse of this bijection is denoted as "log" and can be described by using the Taylor series expansion for logarithm:

$$
\log \eta=-\sum_{\ell=1}^{\infty} \frac{1}{\ell}(\varepsilon-\eta)^{\ell}, \quad \text { for } \eta \in L(\mathcal{B}, \mathbb{C}) \text { with } \eta\left(1_{\mathcal{B}}\right)=1 .
$$

By adjusting the familiar argument for the exponential of a sum of two matrices, one finds that

$$
\begin{array}{ll}
\exp \left(\xi_{1}+\xi_{2}\right)=\exp \left(\xi_{1}\right) \exp \left(\xi_{2}\right), \quad \forall \xi_{1}, \xi_{2} \in L(\mathcal{B}, \mathbb{C}) & \text { such that } \xi_{1}\left(1_{\mathcal{B}}\right)=\xi_{2}\left(1_{\mathcal{B}}\right)=0 \\
& \text { and such that } \xi_{1} \xi_{2}=\xi_{2} \xi_{1}
\end{array}
$$


As a consequence, in the opposite direction of the exp/log bijection one finds that

$$
\begin{array}{ll}
\log \left(\eta_{1} \eta_{2}\right)=\log \left(\eta_{1}\right)+\log \left(\eta_{2}\right), \quad \forall \eta_{1}, \eta_{2} \in L(\mathcal{B}, \mathbb{C}) & \text { such that } \eta_{1}\left(1_{\mathcal{B}}\right)=\eta_{2}\left(1_{\mathcal{B}}\right)=1 \\
& \text { and such that } \eta_{1} \eta_{2}=\eta_{2} \eta_{1}
\end{array}
$$

Finally, let us record here that one can also adjust to our current framework the wellknown exp/log correspondence between derivations and homomorphisms from Lie algebra theory. Let $\mathbb{X}(\mathcal{B})$ be the group of characters of $\mathcal{B}$ from the preceding remark, and on the other hand let us consider the set $\mathbb{I}(\mathcal{B})$ of infinitesimal characters (sometimes also called $\varepsilon$-derivations) of $\mathcal{B}$,

$$
\mathbb{I}(\mathcal{B}):=\left\{\xi \in L(\mathcal{B}, \mathbb{C}) \mid \xi\left(b b^{\prime}\right)=\xi(b) \varepsilon\left(b^{\prime}\right)+\varepsilon(b) \xi\left(b^{\prime}\right), \quad \forall b, b^{\prime} \in \mathcal{B}\right\} .
$$

Then $\mathbb{X}(\mathcal{B})$ is contained in the domain of the log map discussed above, $\mathbb{I}(\mathcal{B})$ is contained in the domain of the exp map, and the two sets correspond to each other in the sense that one has $\log (\mathbb{X}(\mathcal{B}))=\mathbb{I}(\mathcal{B})$. For a discussion of this fact, see for instance Section 4 of the survey paper [5].

\section{The Hopf algebra $\mathcal{Y}^{(k)}$}

Throughout this section we fix a positive integer $k$. Same as in the introduction, we use the notation $\mathcal{Y}^{(k)}$ for the commutative algebra of polynomials

$$
\mathcal{Y}^{(k)}:=\mathbb{C}\left[Y_{w}\left|w \in[k]^{*},\right| w \mid \geq 2\right] .
$$

In addition to that, we will also use the following conventions of notation.

Notation 3.1. $1^{o}$ For a word $w \in[k]^{*}$ such that $|w|=1$ (i.e. such that $w=(i)$ for some $1 \leq i \leq k$ ) we put $Y_{w}:=1$ (the unit of $\mathcal{Y}^{(k)}$ ).

$2^{o}$ Let $w$ be a word in $[k]^{*}$ with $|w|=n \geq 1$, and let $\pi=\left\{A_{1}, \ldots, A_{q}\right\}$ be a partition in $N C(n)$. We will denote

$$
Y_{w ; \pi}:=Y_{w_{1}} \cdots Y_{w_{q}} \in \mathcal{Y}^{(k)}
$$

where $w_{j}=w \mid A_{j}$ for $1 \leq j \leq q$ (and where the restriction $w \mid A$ of the word $w$ to a nonempty subset $A \subseteq\{1, \ldots, n\}$ is defined in the same way as in Equation (2.13) of Definition 2.4).

The comultiplication and counit of $\mathcal{Y}^{(k)}$ are defined as follows.

Definition 3.2. $1^{o}$ Let $\Delta: \mathcal{Y}^{(k)} \rightarrow \mathcal{Y}^{(k)} \otimes \mathcal{Y}^{(k)}$ be the unital algebra homomorphism uniquely determined by the requirement that for every $w \in[k]^{*}$ with $|w|=n \geq 2$ we have

$$
\Delta\left(Y_{w}\right)=\sum_{\pi \in N C(n)} Y_{w ; \pi} \otimes Y_{w ; K(\pi)},
$$

where we use the conventions of notation introduced above (cf. Equation (3.1)), and where $K(\pi)$ is the Kreweras complement of a partition $\pi \in N C(n)$.

$2^{o}$ Let $\varepsilon: \mathcal{Y}^{(k)} \rightarrow \mathbb{C}$ be the unital algebra homomorphism uniquely determined by the requirement that

$$
\varepsilon\left(Y_{w}\right)=0, \quad \forall w \in[k]^{*} \text { with }|w| \geq 2 .
$$


We will verify that the maps $\Delta$ and $\varepsilon$ defined above give indeed a coalgebra (hence bialgebra) structure on $\mathcal{Y}^{(k)}$. The only point that is somewhat laborious is the verification of the coassociativity for $\Delta$, which is covered by the next two lemmas.

Lemma 3.3. Let $w$ be a word in $[k]^{*}$ with $|w|=: n \geq 2$. Let $\rho$ be a partition in $N C(n)$, and consider the element $Y_{w ; \rho} \in \mathcal{Y}^{(k)}$ introduced in Notation 3.1.2. We have

$$
\Delta\left(Y_{w ; \rho}\right)=\sum_{\substack{\pi \in N C(n), \pi \leq \rho}} Y_{w ; \pi} \otimes Y_{w ; K_{\rho}(\pi)},
$$

where $K_{\rho}(\pi)$ denotes the relative Kreweras complement of $\pi$ in $\rho$ (as reviewed in Notation 2.1.3).

Proof. Let us write explicitly $\rho=\left\{B_{1}, \ldots, B_{q}\right\}$ and let us denote $w_{j}:=w \mid B_{j}$ for $1 \leq j \leq q$. Then $Y_{w ; \rho}=Y_{w_{1}} \cdots Y_{w_{q}}$, hence

$$
\begin{aligned}
\Delta\left(Y_{w ; \rho}\right)=\Delta\left(Y_{w_{1}}\right) \cdots \Delta\left(Y_{w_{q}}\right)=\prod_{j=1}^{q}\left(\sum_{\pi_{j} \in N C\left(\left|B_{j}\right|\right)} Y_{w_{j} ; \pi_{j}} \otimes Y_{w_{j} ; K\left(\pi_{j}\right)}\right), \\
=\sum_{\substack{\pi_{1} \in N C\left(\left|B_{1}\right|\right), \ldots \\
\ldots, \pi_{q} \in N C\left(\left|B_{q}\right|\right)}}\left(\prod_{j=1}^{q} Y_{w_{j} ; \pi_{j}}\right) \otimes\left(\prod_{j=1}^{q} Y_{w_{j} ; K\left(\pi_{j}\right)}\right),
\end{aligned}
$$

where the Kreweras complements $K\left(\pi_{j}\right)$ in the latter expressions are taken in the lattices $N C\left(\left|B_{j}\right|\right), 1 \leq j \leq q$.

Now let us consider the bijection (2.4) from Remark 2.2.2. It is immediate that if $\pi \leftrightarrow\left(\pi_{1}, \ldots, \pi_{q}\right)$ via this bijection, then

$$
Y_{w ; \pi}=Y_{w_{1} ; \pi_{1}} \cdots Y_{w_{q} ; \pi_{q}}
$$

Moreover, it was noted in the same Remark 2.2.2 that if $\pi \leftrightarrow\left(\pi_{1}, \ldots, \pi_{q}\right)$ then $K_{\rho}(\pi) \leftrightarrow$ $\left(K\left(\pi_{1}\right), \ldots, K\left(\pi_{q}\right)\right)$, hence in this situation we also have that

$$
Y_{w ; K_{\rho}(\pi)}=Y_{w_{1} ; K\left(\pi_{1}\right)} \cdots Y_{w_{q} ; K\left(\pi_{q}\right)} .
$$

Thus when in (3.5) we perform the change of variable given by the bijection from (2.4), we arrive precisely to the right-hand side of (3.4), as required.

Lemma 3.4. The map $\Delta$ from Definition 3.2.1 is coassociative.

Proof. It suffices to check that $(\Delta \otimes$ id) $\circ \Delta$ and (id $\otimes \Delta) \circ \Delta$ agree on every generator $Y_{w}$, with $w \in[k]^{*}$ such that $|w| \geq 2$. So let us fix such a $w$, and let us denote $|w|=n$. On the one hand we have

$$
\begin{gathered}
((\Delta \otimes \mathrm{id}) \circ \Delta)\left(Y_{w}\right)=(\Delta \otimes \mathrm{id})\left(\sum_{\rho \in N C(n)} Y_{w ; \rho} \otimes Y_{w ; K(\rho)}\right) \\
=\sum_{\rho \in N C(n)}\left(\sum_{\substack{\pi \in N C(n), \pi \leq \rho}} Y_{w ; \pi} \otimes Y_{w ; K_{\rho}(\pi)}\right) \otimes Y_{w ; K(\rho)} \quad \text { (by Lemma 3.3) }
\end{gathered}
$$




$$
=\sum_{\substack{\pi, \rho \in N C(n), \pi \leq \rho}} Y_{w ; \pi} \otimes Y_{w ; K_{\rho}(\pi)} \otimes Y_{w ; K(\rho)} .
$$

On the other hand:

$$
\begin{gathered}
((\mathrm{id} \otimes \Delta) \circ \Delta)\left(Y_{w}\right)=(\mathrm{id} \otimes \Delta)\left(\sum_{\pi \in N C(n)} Y_{w ; \pi} \otimes Y_{w ; K(\pi)}\right) \\
=\sum_{\pi \in N C(n)} Y_{w ; \pi} \otimes\left(\sum_{\substack{\sigma \in N C(n), \sigma \leq K(\pi)}} Y_{w ; \sigma} \otimes Y_{w ; K_{K(\pi)}(\sigma)}\right) \quad \text { (by Lemma 3.3) } \\
=\sum_{\substack{\pi, \sigma \in N C(n), \sigma \leq K(\pi)}} Y_{w ; \pi} \otimes Y_{w ; \sigma} \otimes Y_{w ; K_{K(\pi)}(\sigma)} .
\end{gathered}
$$

Let us now observe that the combinatorial structures which index the sums from (3.6) and (3.7) are precisely those appearing in the bijection (2.10) of Remark 2.2.3. Moreover, we see (by also taking Equation (2.9) into account) that the said bijection (2.10) produces a term-by-term identification of the sums (3.6) and (3.7); hence $(\Delta \otimes \mathrm{id}) \circ \Delta$ and (id $\otimes \Delta) \circ \Delta$ agree on $Y_{w}$, as we wanted.

On $\mathcal{Y}^{(k)}$ we will also consider a grading, which is defined such that every generator $Y_{w}$ of $\mathcal{Y}^{(k)}$ gets to be homogeneous of degree $|w|-1$. More precisely, the homogeneous subspaces $\mathcal{Y}_{n}^{(k)}$ of $\mathcal{Y}^{(k)}$ are defined as follows.

Notation 3.5. For every $n \geq 0$ we denote

$$
\mathcal{Y}_{n}^{(k)}:=\operatorname{span}\left\{\begin{array}{l|l}
Y_{w_{1}} \cdots Y_{w_{q}} & \begin{array}{l}
q \geq 1, w_{1}, \ldots, w_{q} \in[k]^{*} \text { with } \\
\left|w_{1}\right|, \ldots,\left|w_{q}\right| \geq 1 \text { and }\left|w_{1}\right|+\cdots+\left|w_{q}\right|=n+q
\end{array}
\end{array}\right\} .
$$

Proposition 3.6. With the comultiplication, counit and grading defined above, $\mathcal{Y}^{(k)}$ becomes a graded connected Hopf algebra.

Proof. The coassociativity of the comultiplication $\Delta$ was proved in Lemma 3.4, Let us also verify that $\varepsilon$ and $\Delta$ satisfy the counit condition. Clearly, it suffices to check that for every $w \in[k]^{*}$ with $|w| \geq 2$ we have

$$
((\varepsilon \otimes \mathrm{id}) \circ \Delta)\left(Y_{w}\right)=Y_{w}=((\mathrm{id} \otimes \varepsilon) \circ \Delta)\left(Y_{w}\right) .
$$

For such a word $w$ observe first (directly from how $\varepsilon$ is defined) that

$$
\varepsilon\left(Y_{w ; \pi}\right)=0, \quad \forall n \geq 2, \forall \pi \in N C(n) \backslash\left\{0_{n}\right\} .
$$

But then:

$$
\begin{gathered}
((\varepsilon \otimes \mathrm{id}) \circ \Delta)\left(Y_{w}\right)=(\varepsilon \otimes \mathrm{id})\left(\sum_{\pi \in N C(n)} Y_{w ; \pi} \otimes Y_{w ; K(\pi)}\right) \\
=\sum_{\pi \in N C(n)} \varepsilon\left(Y_{w ; \pi}\right) \cdot Y_{w ; K(\pi)}=\varepsilon\left(Y_{w ; 0_{n}}\right) \cdot Y_{w ; 1_{n}}=Y_{w} .
\end{gathered}
$$


The verification that $((\mathrm{id} \otimes \varepsilon) \circ \Delta)\left(Y_{w}\right)=Y_{w}$ is analogous.

We next verify the conditions pertaining to the grading of $\mathcal{Y}^{(k)}$. It is immediate that the subspaces $\mathcal{Y}_{n}^{(k)}(n \geq 0)$ defined by Equation (3.8) give a direct sum decomposition of $\mathcal{Y}^{(k)}$, and satisfy the multiplication relation

$$
\mathcal{Y}_{m}^{(k)} \cdot \mathcal{Y}_{n}^{(k)} \subseteq \mathcal{Y}_{m+n}^{(k)}, \quad \forall m, n \geq 0 .
$$

We have to prove that they also satisfy the comultiplication relation

$$
\Delta\left(\mathcal{Y}_{n}^{(k)}\right) \subseteq \oplus_{m=0}^{n} \mathcal{Y}_{m}^{(k)} \otimes \mathcal{Y}_{n-m}^{(k)}, \quad \forall n \geq 0
$$

By taking into account the specifics of (3.8) and (3.9), we immediately see it is in fact sufficient to verify that for every $w \in[k]^{*}$ with $|w| \geq 2$ we have

$$
\Delta\left(Y_{w}\right) \in \oplus_{m=0}^{|w|-1} \mathcal{Y}_{m}^{(k)} \otimes \mathcal{Y}_{|w|-1-m}^{(k)}
$$

And indeed, (3.11) follows from the obvious implication

$$
\left\{\begin{array}{c}
w \in[k]^{*} \text { with }|w|=n \geq 2 \\
\pi \in N C(n)
\end{array}\right\} \Rightarrow Y_{w ; \pi} \otimes Y_{w ; K(\pi)} \in \mathcal{Y}_{n-|\pi|}^{(k)} \otimes \mathcal{Y}_{n-|K(\pi)|}^{(k)},
$$

combined with the fact that for every $\pi \in N C(n)$ we have $(n-|\pi|)+(n-|K(\pi)|)=n-1$. (For the latter fact we invoke the particular case $\rho=1_{n}$ of Equation (2.3) in Remark 2.2.1.)

Since from (3.8) it is clear that $\mathcal{Y}_{0}^{(k)}=\mathbb{C} \cdot 1$, we have thus proved that $\mathcal{Y}^{(k)}$ is a graded connected bialgebra. As reviewed in Remark 2.10, it then automatically follows that $\mathcal{Y}^{(k)}$ is a Hopf algebra.

We conclude this section by looking at the convolution of characters of $\mathcal{Y}^{(k)}$, and by observing the group isomorphism stated in Theorem 1.2 from the introduction.

Proposition 3.7. For every distribution $\mu \in \mathcal{G}_{k}$, let the character $\chi_{\mu}$ of $\mathcal{Y}^{(k)}$ be as in Definition 1.1. Then the map

$$
\mathcal{G}_{k} \ni \mu \mapsto \chi_{\mu} \in \mathbb{X}\left(\mathcal{Y}^{(k)}\right)
$$

is a group isomorphism, where $\mathcal{G}_{k}$ is endowed with the operation $\otimes$ while $\mathbb{X}\left(\mathcal{Y}^{(k)}\right)$ is endowed with the operation of convolution.

Proof. It is immediately verified that, for every character $\xi \in \mathbb{X}\left(\mathcal{Y}^{(k)}\right)$, the series

$$
\sum_{i=1}^{k} z_{i}+\sum_{\substack{w \in[k]^{*},|w| \geq 2}} \xi\left(Y_{w}\right) z_{w}
$$

appears as $R$-transform for a uniquely determined distribution $\mu \in \mathcal{G}_{k}$. This implies that the map (3.12) is a bijection.

In order to verify that the map (3.12) is a group homomorphism, we fix two distributions $\mu, \nu \in \mathcal{G}_{k}$ for which we prove that

$$
\chi_{\mu \bowtie \nu}=\chi_{\mu} \chi_{\nu}
$$


Clearly, it suffices to prove that the characters appearing on the two sides of (3.13) agree on $Y_{w}$ for every $w \in[k]^{*}$ with $|w| \geq 2$. So we fix such a $w$, we denote $|w|=: n$, and we compute:

$$
\begin{aligned}
\chi_{\mu \boxplus \nu}\left(Y_{w}\right) & \left.=\mathrm{Cf}_{w}\left(R_{\mu \boxplus \nu}\right) \quad \text { (by the definition of } \chi_{\mu \boxplus \nu}\right) \\
& =\sum_{\pi \in N C(n)} \mathrm{Cf}_{w ; \pi}\left(R_{\mu}\right) \cdot \mathrm{Cf}_{w ; K(\pi)}\left(R_{\nu}\right) \quad \text { (by Proposition 2.7) } \\
& \left.=\sum_{\pi \in N C(n)} \chi_{\mu}\left(Y_{w ; \pi}\right) \cdot \chi_{\nu}\left(Y_{w ; K(\pi)}\right) \text { (by definition of } \chi_{\mu}, \chi_{\nu}\right) \\
& =\left(\chi_{\mu} \chi_{\nu}\right)\left(Y_{w}\right),
\end{aligned}
$$

where at the last equality sign of the above calculation we used the formula (2.19) for a convolution product, combined with the formula (3.2) for $\Delta\left(Y_{w}\right)$.

\section{Combinatorial description of $\log \chi_{\mu}$}

In this section we continue to fix a positive integer $k$ and to look at the Hopf algebra $\mathcal{Y}^{(k)}$. The goal of the section is to prove Theorem 1.6 from the introduction, which gives a direct combinatorial description for how the infinitesimal character $\log \chi_{\mu}$ acts on the generators of $\mathcal{Y}^{(k)}$.

Definition 4.1. Let $n$ be a positive integer. A multi-chain in the lattice $N C(n)$ is a tuple of the form

$$
\Gamma=\left(\pi_{0}, \pi_{1}, \ldots, \pi_{\ell}\right)
$$

with $\pi_{0}, \pi_{1}, \ldots, \pi_{\ell} \in N C(n)$ such that $0_{n}=\pi_{0} \leq \pi_{1} \leq \cdots \leq \pi_{\ell}=1_{n}$. The positive integer $\ell$ appearing in (4.1) is called the length of the multi-chain, and is denoted by $|\Gamma|$. If in (4.1) we have $\pi_{j-1} \neq \pi_{j}$ for every $1 \leq j \leq \ell$, then we say that $\Gamma$ is a chain in $N C(n)$.

In our study of $\mathcal{Y}^{(k)}$, multi-chains of non-crossing partitions enter the picture when we consider the iterates $\Delta^{\ell}: \mathcal{Y}^{(k)} \rightarrow\left(\mathcal{Y}^{(k)}\right)^{\otimes \ell}$ of the comultiplication.

Proposition 4.2. Let $w$ be a word in $[k]^{*}$ with $|w|=: n \geq 2$. Then for every positive integer $\ell$ we have

$$
\Delta^{\ell}\left(Y_{w}\right)=\sum_{\substack{\Gamma=\left(\pi_{0}, \pi_{1}, \ldots, \pi_{\ell}\right) \\ \text { multi-chain in } N C(n)}} Y_{w ; K_{\pi_{1}}\left(\pi_{0}\right)} \otimes Y_{w ; K_{\pi_{2}}\left(\pi_{1}\right)} \otimes \cdots \otimes Y_{w ; K_{\pi_{\ell}}\left(\pi_{\ell-1}\right)} .
$$

Proof. By induction on $\ell$. For $\ell=1$ the only multi-chain of length 1 in $N C(n)$ is $\left(0_{n}, 1_{n}\right)$, hence the sum on the right-hand side of (4.2) has only one term, equal to $Y_{w}$. Since $\Delta^{1}$ is by definition the identity map on $\mathcal{Y}^{(k)}$, we see that (4.2) does hold in this case. For $\ell=2$ we have that $\Delta^{2}=\Delta$, while the right-hand side of (4.2) immediately reduces to the summation (3.2) used to define $\Delta$ in Section 3. Hence the formula (4.2) does hold in this case as well. 
Let us now verify the induction step " $\ell \Rightarrow \ell+1$ ", where $\ell$ is a fixed integer, $\ell \geq 2$. We have that $\Delta^{\ell+1}\left(Y_{w}\right)=(\Delta \otimes \underbrace{\mathrm{id} \otimes \cdots \otimes \text { id }}_{\ell-1})\left(\Delta^{\ell}\left(Y_{w}\right)\right)$ (cf. Equation (2.17) of Notation 2.8). In this expression we replace $\Delta^{\ell}\left(Y_{w}\right)$ by using the induction hypothesis; we obtain that

$$
\Delta^{\ell+1}\left(Y_{w}\right)=\sum_{\substack{\Gamma=\left(\pi_{0}, \pi_{1}, \ldots, \pi_{\ell}\right) \\ \text { multi-chain in } N C(n)}} \Delta\left(Y_{w ; \pi_{1}}\right) \otimes Y_{w ; K_{\pi_{2}}\left(\pi_{1}\right)} \otimes \cdots \otimes Y_{w ; K_{\pi_{\ell}}\left(\pi_{\ell-1}\right)},
$$

where on the right-hand side of (4.3) we also used the fact that $K_{\pi_{1}}\left(\pi_{0}\right)=K_{\pi_{1}}\left(0_{n}\right)=\pi_{1}$. In the latter expression we then invoke the explicit formula for $\Delta\left(Y_{w ; \pi_{1}}\right)$ provided by Lemma 3.3. We obtain that $\Delta^{\ell+1}\left(Y_{w}\right)$ is equal to

$$
\sum_{\substack{\Gamma=\left(\pi_{0}, \pi_{1}, \ldots, \pi_{\ell}\right) \\ \text { multi-chain in } N C(n)}}\left(\sum_{\substack{\pi \in N C(n), \pi \leq \pi_{1}}} Y_{w ; \pi} \otimes Y_{w ; K_{\pi_{1}}(\pi)}\right) \otimes Y_{w ; K_{\pi_{2}}\left(\pi_{1}\right)} \otimes \cdots \otimes Y_{w ; K_{\pi_{\ell}}\left(\pi_{\ell-1}\right)},
$$

hence to

$$
\sum_{\substack{\Gamma^{\prime}=\left(\pi_{0}, \pi, \pi_{1}, \ldots, \pi_{\ell}\right) \\ \text { multi-chain in } N C(n)}} Y_{w ; K_{\pi}\left(\pi_{0}\right)} \otimes Y_{w ; K_{\pi_{1}}(\pi)} \otimes Y_{w ; K_{\pi_{2}}\left(\pi_{1}\right)} \otimes \cdots \otimes Y_{w ; K_{\pi_{\ell}}\left(\pi_{\ell-1}\right),}
$$

and this completes the proof of the induction step.

We now move towards proving the explicit formula for the coefficients of the $L S$ transform that was stated in Theorem 1.6. We will use "generalized coefficients" indexed by multi-chains, which are defined as follows.

Definition 4.3. Let $w \in[k]^{*}$ be a word of length $|w|=n \geq 1$, let $\Gamma=\left(\pi_{0}, \pi_{1}, \ldots, \pi_{\ell}\right)$ be a multi-chain in $N C(n)$, and let $f$ be a series in $\mathbb{C}_{0}\left\langle\left\langle z_{1}, \ldots, z_{k}\right\rangle\right\rangle$. The generalized coefficient of $f$ corresponding to $w$ and $\Gamma$ is

$$
\mathrm{Cf}_{w}^{(\Gamma)}(f):=\prod_{j=1}^{\ell} \mathrm{Cf}_{w ; K_{\pi_{j}}\left(\pi_{j-1}\right)}(f) .
$$

A concrete example: say that $n=4$ and that $\Gamma=\left(\pi_{0}, \pi_{1}, \pi_{2}, \pi_{3}\right)$, where $\pi_{0}=0_{4}$, $\pi_{3}=1_{4}$, and $\pi_{1}=\{\{1,3\},\{2\},\{4\}\}, \pi_{2}=\{\{1,3,4\},\{2\}\}$. Then we have

$$
\left\{\begin{array}{l}
K_{\pi_{1}}\left(\pi_{0}\right)=\pi_{1}=\{\{1,3\},\{2\},\{4\}\} \\
K_{\pi_{2}}\left(\pi_{1}\right)=\{\{1\},\{2\},\{3,4\}\} \\
K_{\pi_{3}}\left(\pi_{2}\right)=K\left(\pi_{2}\right)=\{\{1,2\},\{3\},\{4\}\},
\end{array}\right.
$$

hence for a word $w=\left(i_{1}, i_{2}, i_{3}, i_{4}\right) \in[k]^{*}$ and a series $f \in \mathbb{C}_{0}\left\langle\left\langle z_{1}, \ldots, z_{k}\right\rangle\right\rangle$ we get that

$$
\begin{gathered}
\mathrm{Cf}_{w}^{(\Gamma)}(f)=\left(\mathrm{Cf}_{\left(i_{1}, i_{3}\right)}(f) \mathrm{Cf}_{\left(i_{2}\right)}(f) \mathrm{Cf}_{\left(i_{4}\right)}(f)\right) . \\
\left(\mathrm{Cf}_{\left(i_{1}\right)}(f) \mathrm{Cf}_{\left(i_{2}\right)}(f) \mathrm{Cf}_{\left(i_{3}, i_{4}\right)}(f)\right) \cdot\left(\mathrm{Cf}_{\left(i_{1}, i_{2}\right)}(f) \mathrm{Cf}_{\left(i_{3}\right)}(f) \mathrm{Cf}_{\left(i_{4}\right)}(f)\right) .
\end{gathered}
$$

Note that if $f$ happens to be an $R$-transform $R_{\mu}$ denoted as in Equation (1.6) of the introduction, then the above formula simplifies to

$$
\mathrm{Cf}_{w}^{(\Gamma)}(f)=\alpha_{\left(i_{1}, i_{3}\right)} \cdot \alpha_{\left(i_{3}, i_{4}\right)} \cdot \alpha_{\left(i_{1}, i_{2}\right)} .
$$


The generalized coefficients defined above come into our calculations with characters of $\mathcal{Y}^{(k)}$ via the following lemma.

Lemma 4.4. Let $\mu$ be a distribution in $\mathcal{G}_{k}$, let $w$ be a word in $[k]^{*}$ with $|w|=n \geq 2$ and let $\ell$ be a positive integer. If $\ell<n$ then

$$
\left(\chi_{\mu}-\varepsilon\right)^{\ell}\left(Y_{w}\right)=\sum_{\substack{\Gamma \text { chain } \\ \text { in } N C(n),|\Gamma|=\ell}} C f_{w}^{(\Gamma)}\left(R_{\mu}\right) .
$$

If $\ell \geq n$, then $\left(\chi_{\mu}-\varepsilon\right)^{\ell}\left(Y_{w}\right)=0$.

Proof. We evaluate $\left(\chi_{\mu}-\varepsilon\right)^{\ell}\left(Y_{w}\right)$ by using Equation (2.20) and then by replacing $\Delta^{\ell}\left(Y_{w}\right)$ from Proposition 4.2. In this way we arrive to a summation over multi-chains,

$$
\left(\chi_{\mu}-\varepsilon\right)^{\ell}\left(Y_{w}\right)=\sum_{\substack{\Gamma \text { multi-chain } \\ \text { in } N C(n),|\Gamma|=\ell}} \operatorname{term}_{\Gamma}
$$

where the term of the sum indexed by the multi-chain $\Gamma=\left(\pi_{0}, \pi_{1}, \ldots, \pi_{\ell}\right)$ is

$$
\operatorname{term}_{\Gamma}:=\prod_{j=1}^{\ell}\left(\chi_{\mu}-\varepsilon\right)\left(Y_{w ; K_{\pi_{j}}\left(\pi_{j-1}\right)}\right) .
$$

Note that if in (4.7) there exists an index $j$ such that $\pi_{j-1}=\pi_{j}$, then it follows that

$$
\left(\chi_{\mu}-\varepsilon\right)\left(Y_{w ; K_{\pi_{j}}}\left(\pi_{j-1}\right)\right)=\left(\chi_{\mu}-\varepsilon\right)\left(Y_{w ; 0_{n}}\right)=\left(\chi_{\mu}-\varepsilon\right)(1)=1-1=0 .
$$

This observation shows that term ${ }_{\Gamma}$ vanishes whenever $\Gamma$ is not a chain.

Since the biggest possible length of a chain in $N C(n)$ is $n-1$, the above observation implies that for $\ell \geq n$ all the terms of the sum on the right-hand side of (4.6) are equal to 0 . Hence for $\ell \geq n$ we have that $\left(\chi_{\mu}-\varepsilon\right)^{\ell}\left(Y_{w}\right)=0$.

Finally, let us assume that $\ell<n$ and let us look at term ${ }_{\Gamma}$ in the case when $\Gamma=$ $\left(\pi_{0}, \pi_{1}, \ldots, \pi_{\ell}\right)$ is a chain. In this case every relative Kreweras complement $K_{\pi_{j}}\left(\pi_{j-1}\right)$ $(1 \leq j \leq \ell)$ is different from $0_{n}$; this immediately implies that $\varepsilon\left(Y_{w ; K_{\pi_{j}}}\left(\pi_{j-1}\right)\right)=0$, hence that

$$
\left(\chi_{\mu}-\varepsilon\right)\left(Y_{w ; K_{\pi_{j}}\left(\pi_{j-1}\right)}\right)=\chi_{\mu}\left(Y_{w ; K_{\pi_{j}}\left(\pi_{j-1}\right)}\right)=\mathrm{Cf}_{w ; K_{\pi_{j}}\left(\pi_{j-1}\right)}\left(R_{\mu}\right)
$$

Thus if $\Gamma$ is a chain, then the right-hand side of (4.7) is equal to $\mathrm{Cf}_{w}^{(\Gamma)}\left(R_{\mu}\right)$, and (4.5) follows.

As a consequence, we obtain the explicit formula for $\left(\log \chi_{\mu}\right)\left(Y_{w}\right)$ that was announced in the introduction of the paper.

Proposition 4.5. Let $\mu$ be a distribution in $\mathcal{G}_{k}$, and let $w$ be a word in $[k]^{*}$, with $|w|=$ $n \geq 2$. Then

$$
\left(\log \chi_{\mu}\right)\left(Y_{w}\right)=\sum_{\substack{\Gamma \text { chain } \\ \text { in } N C(n)}} \frac{(-1)^{1+|\Gamma|}}{|\Gamma|} C f_{w}^{(\Gamma)}\left(R_{\mu}\right)
$$


Proof. This follows immediately when we combine the formal series expansion which defines $\log \chi_{\mu}$ (Equation (2.26) in Remark 2.12) with the formula obtained in Lemma 4.4.

Example 4.6. Let us see how the expression on the right-hand side of Equation (4.8) concretely looks, for some small values of $|w|$.

Case $|w|=2$. There exists a unique chain in $N C(2)$, namely $\Gamma=\left(0_{2}, 1_{2}\right)$. Thus for a word of length $2, w=\left(i_{1}, i_{2}\right)$, the formula (4.8) simply amounts to

$$
\mathrm{Cf}_{\left(i_{1}, i_{2}\right)}\left(L S_{\mu}\right)=\mathrm{Cf}_{\left(i_{1}, i_{2}\right)}\left(R_{\mu}\right) .
$$

Case $|w|=3$. The lattice $N C(3)$ has 1 chain of length $1, \Gamma=\left(0_{3}, 1_{3}\right)$, and has 3 chains of length $2: \Gamma_{i}=\left(0_{3}, \pi_{i}, 1_{3}\right), 1 \leq i \leq 3$, where we put

$$
\pi_{1}=\{\{1\},\{2,3\}\}, \pi_{2}=\{\{1,3\},\{2\}\}, \pi_{3}=\{\{1,2\},\{3\}\} .
$$

The Kreweras complements of $\pi_{1}, \pi_{2}, \pi_{3}$ are $\pi_{2}, \pi_{3}$ and $\pi_{1}$, respectively. Thus for a word of length $3, w=\left(i_{1}, i_{2}, i_{3}\right)$, we have

$$
\begin{aligned}
\mathrm{Cf}_{w}^{\left(\Gamma_{1}\right)}\left(R_{\mu}\right) & =\mathrm{Cf}_{w ; \pi_{1}}\left(R_{\mu}\right) \cdot \mathrm{Cf}_{w ; \pi_{2}}\left(R_{\mu}\right) \\
& =\left(\mathrm{Cf}_{\left(i_{1}, i_{2}\right)}\left(R_{\mu}\right) \mathrm{Cf}_{\left(i_{3}\right)}\left(R_{\mu}\right)\right) \cdot\left(\mathrm{Cf}_{\left(i_{1}, i_{3}\right)}\left(R_{\mu}\right) \mathrm{Cf}_{\left(i_{2}\right)}\left(R_{\mu}\right)\right),
\end{aligned}
$$

and similar formulas hold for $\mathrm{Cf}_{w}^{\left(\Gamma_{2}\right)}\left(R_{\mu}\right)$ and $\mathrm{Cf}_{w}^{\left(\Gamma_{3}\right)}\left(R_{\mu}\right)$. By taking into account that the linear coefficients of $R_{\mu}$ are all equal to 1 , we thus get that

$$
\begin{gathered}
\mathrm{Cf}_{\left(i_{1}, i_{2}, i_{3}\right)}\left(L S_{\mu}\right)=\mathrm{Cf}_{\left(i_{1}, i_{2}, i_{3}\right)}\left(R_{\mu}\right)-\frac{1}{2} \mathrm{Cf}_{\left(i_{1}, i_{2}\right)}\left(R_{\mu}\right) \mathrm{Cf}_{\left(i_{1}, i_{3}\right)}\left(R_{\mu}\right) \\
-\frac{1}{2} \mathrm{Cf}_{\left(i_{1}, i_{3}\right)}\left(R_{\mu}\right) \mathrm{Cf}_{\left(i_{2}, i_{3}\right)}\left(R_{\mu}\right)-\frac{1}{2} \mathrm{Cf}_{\left(i_{2}, i_{3}\right)}\left(R_{\mu}\right) \mathrm{Cf}_{\left(i_{1}, i_{2}\right)}\left(R_{\mu}\right) .
\end{gathered}
$$

Case $|w|=4$. The lattice $N C(4)$ has 1 chain of length 1 , has 12 chains of length 2 (of the form $\left(0_{4}, \pi, 1_{4}\right)$ with $\pi$ running in $N C(4) \backslash\left\{0_{4}, 1_{4}\right\}$ ), and has 16 chains of length 3 . Some direct (but more tedious) calculations similar to the ones shown above lead to the conclusion that for a word $w=\left(i_{1}, \ldots, i_{4}\right) \in\{1, \ldots, k\}^{4}$ one has

$$
\mathrm{Cf}_{\left(i_{1}, i_{2}, i_{3}, i_{4}\right)}\left(L S_{\mu}\right)=\mathrm{Cf}_{\left(i_{1}, i_{2}, i_{3}, i_{4}\right)}\left(R_{\mu}\right)-\frac{1}{2} \Sigma+\frac{1}{3} \Sigma^{\prime}+\frac{1}{6} \Sigma^{\prime \prime},
$$

where each of $\Sigma, \Sigma^{\prime}$ and $\Sigma^{\prime \prime}$ is a sum of products of coefficients of $R_{\mu}$. (The fraction $1 / 6$ which multiplies $\Sigma^{\prime \prime}$ appears from cancelations, $1 / 6=2 \cdot 1 / 3-1 / 2$.) For the record, we indicate below what these sums are exactly; in order to save space, the next formula has the coefficients of $R_{\mu}$ denoted as in Equation (1.6) of the introduction, by writing " $\alpha_{(\ldots)}$ " instead of " $\mathrm{Cf}_{(\ldots)}\left(R_{\mu}\right)$ ". So, we have:

$$
\begin{gathered}
\Sigma=\alpha_{\left(i_{1}, i_{2}, i_{3}\right)}\left(\alpha_{\left(i_{1}, i_{4}\right)}+\alpha_{\left(i_{3}, i_{4}\right)}\right)+\alpha_{\left(i_{1}, i_{2}, i_{4}\right)}\left(\alpha_{\left(i_{3}, i_{4}\right)}+\alpha_{\left(i_{2}, i_{3}\right)}\right) \\
+\alpha_{\left(i_{1}, i_{3}, i_{4}\right)}\left(\alpha_{\left(i_{2}, i_{3}\right)}+\alpha_{\left(i_{1}, i_{2}\right)}\right)+\alpha_{\left(i_{2}, i_{3}, i_{4}\right)}\left(\alpha_{\left(i_{1}, i_{2}\right)}+\alpha_{\left(i_{1}, i_{4}\right)}\right), \\
\Sigma^{\prime}=\alpha_{\left(i_{1}, i_{2}\right)} \alpha_{\left(i_{1}, i_{3}\right)} \alpha_{\left(i_{1}, i_{4}\right)}+\alpha_{\left(i_{1}, i_{2}\right)} \alpha_{\left(i_{2}, i_{3}\right)} \alpha_{\left(i_{2}, i_{4}\right)}+\alpha_{\left(i_{1}, i_{3}\right)} \alpha_{\left(i_{2}, i_{3}\right)} \alpha_{\left(i_{3}, i_{4}\right)} \\
+\alpha_{\left(i_{1}, i_{4}\right)} \alpha_{\left(i_{2}, i_{4}\right)} \alpha_{\left(i_{3}, i_{4}\right)}+\alpha_{\left(i_{1}, i_{2}\right)} \alpha_{\left(i_{2}, i_{3}\right)} \alpha_{\left(i_{3}, i_{4}\right)}+\alpha_{\left(i_{1}, i_{2}\right)} \alpha_{\left(i_{2}, i_{3}\right)} \alpha_{\left(i_{1}, i_{4}\right)} \\
+\alpha_{\left(i_{1}, i_{2}\right)} \alpha_{\left(i_{1}, i_{4}\right)} \alpha_{\left(i_{3}, i_{4}\right)}+\alpha_{\left(i_{1}, i_{4}\right)} \alpha_{\left(i_{2}, i_{3}\right)} \alpha_{\left(i_{3}, i_{4}\right)},
\end{gathered}
$$

and

$$
\begin{aligned}
& \Sigma^{\prime \prime}=\alpha_{\left(i_{1}, i_{2}\right)} \alpha_{\left(i_{1}, i_{3}\right)} \alpha_{\left(i_{3}, i_{4}\right)}+\alpha_{\left(i_{1}, i_{2}\right)} \alpha_{\left(i_{2}, i_{4}\right)} \alpha_{\left(i_{3}, i_{4}\right)} \\
& \quad+\alpha_{\left(i_{1}, i_{3}\right)} \alpha_{\left(i_{1}, i_{4}\right)} \alpha_{\left(i_{2}, i_{3}\right)}+\alpha_{\left(i_{1}, i_{4}\right)} \alpha_{\left(i_{2}, i_{3}\right)} \alpha_{\left(i_{2}, i_{4}\right)} .
\end{aligned}
$$


We conclude this section with the observation that if one removes the division by $|\Gamma|$ on the right-hand side of (4.8), then what results is a formula for calculating the $R$-transform of an inverse in the group $\left(\mathcal{G}_{k}, \otimes\right)$. This happens because (besides its use in proving Proposition 4.5) Lemma 4.4 can also be used in connection to the inverse formula for characters that was reviewed in (2.24) of Remark 2.11. The precise statement of this fact goes as follows.

Proposition 4.7. Let $\mu$ be a distribution in $\mathcal{G}_{k}$, and let $\nu$ denote the inverse of $\mu$ under the operation $\otimes$. Then for every word $w \in[k]^{*}$ with $|w|=n \geq 2$ we have

$$
C f_{w}\left(R_{\nu}\right)=\sum_{\Gamma \text { chain }}(-1)^{|\Gamma|} C f_{w}^{(\Gamma)}\left(R_{\mu}\right) .
$$

in $N C(n)$

Proof. We know that

$$
\begin{aligned}
\mathrm{Cf}_{w}\left(R_{\nu}\right) & =\chi_{\nu}\left(Y_{w}\right) \quad\left(\text { by definition of } \chi_{\nu}\right) \\
& =\chi_{\mu}^{-1}\left(Y_{w}\right) \quad(\text { by Theorem 1.2) } \\
& \left.=\varepsilon\left(Y_{w}\right)+\sum_{\ell=1}^{\infty}(-1)^{\ell}\left(\chi_{\mu}-\varepsilon\right)^{\ell}\left(Y_{w}\right) \quad(\text { by Equation (2.24) })\right) \\
& =\sum_{\ell=1}^{n-1}(-1)^{\ell}\left(\sum_{\substack{\Gamma \text { chain } \\
\text { in } N C(n),|\Gamma|=\ell}} \mathrm{Cf}_{w}^{(\Gamma)}\left(R_{\mu}\right)\right),
\end{aligned}
$$

where at the last equality sign we used Lemma 4.4 and the fact that $\varepsilon\left(Y_{w}\right)=0$. The formula (4.15) then immediately follows.

\section{Some basic properties of the LS-transform}

Proposition 5.1. Let $(\mathcal{A}, \varphi)$ be a non-commutative probability space. Let a be an element of $\mathcal{A}$ such that $\varphi(a)=1$, and let $\mu_{1} \in \mathcal{G}_{1}$ be the distribution of a. Moreover, let $k$ be a positive integer and let $\mu \in \mathcal{G}_{k}$ denote the distribution of the $k$-tuple $(a, a, \ldots, a)$. Then

$$
L S_{\mu}\left(z_{1}, \ldots, z_{k}\right)=L S_{\mu_{1}}\left(z_{1}+\cdots+z_{k}\right) .
$$

Proof. The $R$-transforms of $\mu$ and of $\mu_{1}$ are related by the formula

$$
R_{\mu}\left(z_{1}, \ldots, z_{k}\right)=R_{\mu_{1}}\left(z_{1}+\cdots+z_{k}\right)
$$

(see Remark 17.13 on p. 280 of [11]). This means that

$$
\mathrm{Cf}_{\left(i_{1}, \ldots, i_{n}\right)}\left(R_{\mu}\right)=\mathrm{Cf}_{(1, \ldots, 1)}\left(R_{\mu_{1}}\right), \quad \forall n \geq 1, \forall 1 \leq i_{1}, \ldots, i_{n} \leq k,
$$

where on the right-hand side of (5.3) (and same for (5.4)-(5.6) below) there are $n$ repetitions of 1 in " $(1, \ldots, 1)$ ". From (5.3) one immediately sees, by simply following definitions, that

$$
\mathrm{Cf}_{\left(i_{1}, \ldots, i_{n}\right) ; \pi}\left(R_{\mu}\right)=\mathrm{Cf}_{(1, \ldots, 1) ; \pi}\left(R_{\mu_{1}}\right), \quad \forall n \geq 1, \forall 1 \leq i_{1}, \ldots, i_{n} \leq k, \forall \pi \in N C(n),
$$


and consequently that

$$
\mathrm{Cf}_{\left(i_{1}, \ldots, i_{n}\right)}^{(\Gamma)}\left(R_{\mu}\right)=\mathrm{Cf}_{(1, \ldots, 1)}^{(\Gamma)}\left(R_{\mu_{1}}\right), \quad \forall n \geq 1, \forall 1 \leq i_{1}, \ldots, i_{n} \leq k, \forall \Gamma,
$$

where in (5.5) $\Gamma$ is an arbitrary chain of partitions from $N C(n)$. By using Equation (5.5) and the expression found in Proposition 4.5 for the coefficients of the $L S$-transform, we obtain that

$$
\mathrm{Cf}_{\left(i_{1}, \ldots, i_{n}\right)}\left(L S_{\mu}\right)=\mathrm{Cf}_{(1, \ldots, 1)}\left(L S_{\mu_{1}}\right), \quad \forall n \geq 2, \forall 1 \leq i_{1}, \ldots, i_{n} \leq k,
$$

and (5.1) follows.

Example 5.2. Here is an example of how the $L S$-transform can be used in the $C^{*}$ framework. Let $(\mathcal{A}, \varphi)$ be a tracial $2 C^{*}$-probability space, let $a_{1}, \ldots, a_{k} \in \mathcal{A}$ be selfadjoint and let $p \in \mathcal{A}$ be positive, such that $p$ is freely independent from $\left\{a_{1}, \ldots, a_{k}\right\}$ and such that $\varphi(p)=\varphi\left(a_{1}\right)=\cdots=\varphi\left(a_{k}\right)=1$. Consider the selfadjoint elements $b_{1}, \ldots, b_{k} \in \mathcal{A}$ defined by $b_{i}=p^{1 / 2} a_{i} p^{1 / 2}, 1 \leq i \leq k$. It is immediate that the $k$-tuple $\left(b_{1}, \ldots, b_{k}\right)$ has the same distribution as $\left(a_{1} p, \ldots, a_{k} p\right)$, and hence that

$$
\mu_{\vec{b}}=\mu_{\vec{a}} \otimes \mu_{\vec{p}}
$$

where $\mu_{\vec{b}}, \mu_{\vec{a}}, \mu_{\vec{p}}$ denote the distributions of the $k$-tuples $\left(b_{1}, \ldots, b_{k}\right),\left(a_{1}, \ldots, a_{k}\right)$ and $(p, \ldots, p)$, respectively. Moreover, it is known that $\mu_{\vec{p}}$ belongs to the centre of $\left(\mathcal{G}_{k}, \nabla\right)$ (see discussion on pp. 277-279 of [11]). Thus Corollary 1.5 applies to this situation, and if we also take Proposition 5.1 into account we arrive to the formula

$$
L S_{\vec{b}}\left(z_{1}, \ldots, z_{k}\right)=L S_{\vec{a}}\left(z_{1}, \ldots, z_{k}\right)+L S_{p}\left(z_{1}+\cdots+z_{k}\right)
$$

where we wrote in short $L S_{\vec{a}}$ and $L S_{\vec{b}}$ for the $L S$-transforms of $\mu_{\vec{a}}$ and $\mu_{\vec{b}}$, and where $L S_{p}$ stands for the $L S$-transform of the (1-dimensional) distribution of the element $p$.

Remark 5.3. Let $(\mathcal{A}, \varphi)$ be a non-commutative probability space, and let $a_{1}, \ldots, a_{k}$ be elements of $\mathcal{A}$ such that $\varphi\left(a_{1}\right)=\cdots=\varphi\left(a_{k}\right)=1$. Let $\mu$ be the distribution of the $k$-tuple $\left(a_{1}, \ldots, a_{k}\right)$; on the other hand, for every $1 \leq i \leq k$ let $\mu_{i}$ denote the distribution of the element $a_{i}$ (thus $\mu \in \mathcal{G}_{k}$, whereas $\mu_{1}, \ldots, \mu_{k} \in \mathcal{G}_{1}$ ). Then for every $1 \leq i \leq k$ we have that

$$
L S_{\mu}\left(0, \ldots, 0, z_{i}, 0, \ldots, 0\right)=L S_{\mu_{i}}\left(z_{i}\right)
$$

The easy proof of (5.9) (which is very similar to the argument shown in the proof of Proposition 5.1) is left to the reader.

We next look at the important special case when (in the same notations as above) $a_{1}, \ldots, a_{k}$ form a freely independent family. Let us recall here a fundamental fact from the theory of the multivariable $R$-transform: the free independence of $a_{1}, \ldots, a_{k}$ is equivalent to the vanishing of the mixed coefficients of $R_{\mu}$, hence to the fact that the series $R_{\mu}\left(z_{1}, \ldots, z_{k}\right)$ separates the variables (see Lecture 16 in [11]). The next proposition shows that this feature of the $R$-transform is passed to the $L S$-transform.

\footnotetext{
${ }^{2}$ The hypothesis that $\varphi$ is a trace isn't in fact necessary for the example. The only place where the traciality of $\varphi$ is used is in order to observe that $\left(b_{1}, \ldots, b_{k}\right)$ has the same distribution as $\left(a_{1} p, \ldots, a_{k} p\right)$. This statement is obvious when $\varphi$ is a trace, but is still true (though less obvious) without assuming that $\varphi$ is a trace - one can derive it solely from the hypothesis that $p$ is free from $\left\{a_{1}, \ldots, a_{k}\right\}$, via a suitable calculation with free cumulants.
} 
Proposition 5.4. Let $(\mathcal{A}, \varphi)$, the elements $a_{1}, \ldots, a_{k} \in \mathcal{A}$, and the distributions $\mu \in \mathcal{G}_{k}$ and $\mu_{1}, \ldots, \mu_{k} \in \mathcal{G}_{1}$ be as in the preceding remark. The following statements are equivalent:

(1) The elements $a_{1}, \ldots, a_{k}$ form a freely independent family in $(\mathcal{A}, \varphi)$.

(2) The $L S$-transform of $\mu$ separates the variables. That is, there exist series $u_{1}, \ldots, u_{k} \in$ $\mathbb{C}[[z]]$ such that

$$
L S_{\mu}\left(z_{1}, \ldots, z_{k}\right)=u_{1}\left(z_{1}\right)+\cdots+u_{k}\left(z_{k}\right)
$$

(3) One has

$$
L S_{\mu}\left(z_{1}, \ldots, z_{k}\right)=L S_{\mu_{1}}\left(z_{1}\right)+\cdots+L S_{\mu_{k}}\left(z_{k}\right) .
$$

Proof. The implication (3) $\Rightarrow$ (2) is trivial, while (2) $\Rightarrow$ (3) follows immediately from Remark 5.3. The bulk of the proof will be devoted to the equivalence of (1) and (2).

"(1) $\Rightarrow(2)$ ". We have to show that all the mixed coefficients of $L S_{\mu}$ vanish. So let us fix $n \geq 2$ and $1 \leq i_{1}, \ldots, i_{n} \leq k$ such that $\operatorname{Cf}_{\left(i_{1}, \ldots, i_{n}\right)}\left(L S_{\mu}\right) \neq 0$; our goal is to prove that $i_{1}=i_{2}=\cdots=i_{n}$.

From the formula for $\mathrm{Cf}_{\left(i_{1}, \ldots, i_{n}\right)}\left(L S_{\mu}\right)$ found in Proposition 4.5 we infer that there must exist chains $\Gamma$ of partitions in $N C(n)$ such that $\mathrm{Cf}_{\left(i_{1}, \ldots, i_{n}\right)}^{(\Gamma)}\left(R_{\mu}\right) \neq 0$. Let us fix such a chain, $\Gamma=\left(\pi_{0}, \pi_{1}, \ldots, \pi_{\ell}\right)$. We have that

$$
0 \neq \mathrm{Cf}_{\left(i_{1}, \ldots, i_{n}\right)}^{(\Gamma)}\left(R_{\mu}\right)=\prod_{j=1}^{k} \mathrm{Cf}_{\left(i_{1}, \ldots, i_{n}\right) ; K_{\pi_{j}}\left(\pi_{j-1}\right)}\left(R_{\mu}\right),
$$

hence that

$$
\mathrm{Cf}_{\left(i_{1}, \ldots, i_{n}\right) ; K_{\pi_{j}}\left(\pi_{j-1}\right)}\left(R_{\mu}\right) \neq 0, \quad \forall 1 \leq j \leq \ell .
$$

At this point it is convenient to encode our $n$-tuple $\left(i_{1}, \ldots, i_{n}\right)$ as a function $I$ : $\{1, \ldots, n\} \rightarrow\{1, \ldots, k\}$, where $I(m):=i_{m}$ for $1 \leq m \leq n$. Then (5.12) can be combined with the fact (equivalent to the hypothesis in (1)) that the mixed coefficients of $R_{\mu}$ are all equal to 0 , in order to infer that the function $I$ is constant along every block of the partition $K_{\pi_{j}}\left(\pi_{j-1}\right)$, for every $1 \leq j \leq \ell$. In view of how the permutation associated to a non-crossing partition is defined (see Notation 2.1,2), the latter fact is equivalent to

$$
I \circ P_{K_{\pi_{j}}\left(\pi_{j-1}\right)}=I, \quad \forall 1 \leq j \leq \ell .
$$

Now, the formula for the permutation associated to a relative Kreweras complement (Equation (2.2) in Notation 2.1,3) gives us that

$$
P_{K_{\pi_{j}}\left(\pi_{j-1}\right)}=P_{\pi_{j-1}}^{-1} P_{\pi_{j}}, \quad \forall 1 \leq j \leq \ell
$$

When we put together (5.13) and (5.14), we find that

$$
\begin{aligned}
I & =I \circ\left(P_{K_{\pi_{1}}\left(\pi_{0}\right)} P_{K_{\pi_{2}}\left(\pi_{1}\right)} \cdots P_{K_{\pi_{\ell}}\left(\pi_{\ell-1}\right)}\right) \\
& =I \circ\left(\left(P_{\pi_{0}}^{-1} P_{\pi_{1}}\right)\left(P_{\pi_{1}}^{-1} P_{\pi_{2}}\right)\left(P_{\pi_{\ell-1}}^{-1} P_{\pi_{\ell}}\right)\right) \\
& =I \circ\left(P_{\pi_{0}}^{-1} P_{\pi_{\ell}}\right) .
\end{aligned}
$$

But $\pi_{0}=0_{n}$ and $\pi_{\ell}=1_{n}$, so the permutation $P_{\pi_{0}}^{-1} P_{\pi_{\ell}}$ is just the cycle $1 \mapsto 2 \mapsto \cdots \mapsto n \mapsto 1$. The fact that $I=I \circ\left(P_{\pi_{0}}^{-1} P_{\pi_{\ell}}\right)$ then immediately implies that $I$ is a constant function, hence that $i_{1}=i_{2}=\cdots=i_{n}$, as we wanted. 
"(2) $\Rightarrow(1)$ ". We will verify the free independence of $a_{1}, \ldots, a_{k}$ by checking that all the mixed coefficients of $R_{\mu}$ vanish:

$$
\left\{\begin{array}{c}
\mathrm{Cf}_{\left(i_{1}, \ldots, i_{n}\right)}\left(R_{\mu}\right)=0 \text { for every } n \geq 2 \text { and every } 1 \leq i_{1}, \ldots, i_{n} \leq k \\
\text { for which it is not true that } i_{1}=i_{2}=\cdots=i_{n} .
\end{array}\right.
$$

The verification of (5.15) will be made by induction on $n$.

For the base case $n=2$ we just have to note that $\mathrm{Cf}_{\left(i_{1}, i_{2}\right)}\left(R_{\mu}\right)=\mathrm{Cf}_{\left(i_{1}, i_{2}\right)}\left(L S_{\mu}\right)=0$ for every $1 \leq i_{1}, i_{2} \leq k$ such that $i_{1} \neq i_{2}$ (where we used Equation (4.9) from Example 4.6, and the hypothesis that the mixed coefficients of $L S_{\mu}$ are all equal to 0 ).

The induction step: we assume that (5.15) was proved for $2,3, \ldots, n-1$, and we prove that it also holds for $n$, where $n \geq 3$. Fix some indices $1 \leq i_{1}, \ldots, i_{n} \leq k$ for which it is not true that $i_{1}=i_{2}=\cdots=i_{n}$. Observe that Equation (4.8) from Proposition 4.5 can be re-written in the form

$$
\mathrm{Cf}_{\left(i_{1}, \ldots, i_{n}\right)}\left(R_{\mu}\right)=\mathrm{Cf}_{\left(i_{1}, \ldots, i_{n}\right)}\left(L S_{\mu}\right)+\sum_{\substack{\Gamma \text { chain in } N C(n) \\ \text { with }|\Gamma| \geq 2}} \frac{(-1)^{|\Gamma|}}{|\Gamma|} \mathrm{Cf}_{\left(i_{1}, \ldots, i_{n}\right)}^{(\Gamma)}\left(R_{\mu}\right) .
$$

Now, if $\Gamma$ is a chain of partitions in $N C(n)$ with $|\Gamma| \geq 2$ then we get that $\mathrm{Cf}_{\left(i_{1}, \ldots, i_{n}\right)}^{(\Gamma)}\left(R_{\mu}\right)=0$ by an argument very similar to the one used in the proof of (1) $\Rightarrow$ (2) (where this time we also use the induction hypothesis, and the fact the explicit writing of $\mathrm{Cf}_{\left(i_{1}, \ldots, i_{n}\right)}^{(\Gamma)}\left(R_{\mu}\right)$ only involves coefficients of order $\leq(n-1)$ of $\left.R_{\mu}\right)$. Since we also know that $\mathrm{Cf}_{\left(i_{1}, \ldots, i_{n}\right)}\left(L S_{\mu}\right)=0$ (by the hypothesis that the mixed coefficients of $L S_{\mu}$ vanish), Equation (5.16) gives us that $\mathrm{Cf}_{\left(i_{1}, \ldots, i_{n}\right)}\left(R_{\mu}\right)=0$, and this completes our inductive argument.

\section{Case of one variable: LS and $\log \mathrm{S}$}

This section is devoted to the one-variable framework: we discuss connections that $\left(\mathcal{G}_{1}, \otimes\right)$ and $\mathcal{Y}^{(1)}$ have with symmetric functions, and we prove Theorem 1.8 .

Remark 6.1. In this remark we make an update of our notations for series and we give a more precise review of what is the $S$-transform.

In the one-variable framework our notations for series are simplified by the fact that we only have to deal with the commutative algebra $\mathbb{C}[[z]]$. Observe for instance that if $\mu$ is a distribution in $\mathcal{G}_{1}$, then the notation used in Equation (1.6) of the introduction for the $R$-transform $R_{\mu}$ now simplifies to just

$$
R_{\mu}(z)=z+\sum_{n=2}^{\infty} \alpha_{n} z^{n} .
$$

In what follows we will work with two kinds of "inverses" for series in $\mathbb{C}[[z]]$. On the one hand we have the inverse under multiplication, which is defined for a series $f(z)=$ $\sum_{n=0}^{\infty} t_{n} z^{n}$ with $t_{0} \neq 0$, and will be denoted as " $1 / f$ ". On the other hand we have the inverse under composition, which is defined for a series $f(z)=\sum_{n=1}^{\infty} t_{n} z^{n}$ with $t_{1} \neq 0$, and will be denoted as " $f\langle-1\rangle$ ". 
The definition of the $S$-transform for a distribution $\mu \in \mathcal{G}_{1}$ goes as follows: one first considers the moment series of $\mu$,

$$
M_{\mu}(z):=z+\sum_{n=2}^{\infty} \mu\left(X^{n}\right) z^{n} \in \mathbb{C}[[z]],
$$

then puts

$$
S_{\mu}(z):=\frac{1+z}{z} M_{\mu}^{\langle-1\rangle}(z) .
$$

In this paper we prefer to write $S_{\mu}$ in terms of the $R$-transform $R_{\mu}$; this is done by a similarly looking formula,

$$
S_{\mu}(z)=\frac{1}{z} R_{\mu}^{\langle-1\rangle}(z)
$$

(see [11], Remark 16.18 on p. 270).

It is clear that for $\mu \in \mathcal{G}_{1}$, the $S$-transform $S_{\mu} \in \mathbb{C}[[z]]$ has constant term equal to 1 . Hence one can consider the inverse under multiplication $1 / S_{\mu}$, and this is another series with constant term equal to 1:

$$
1 / S_{\mu}(z)=1+\sum_{n=1}^{\infty} \gamma_{n} z^{n}
$$

for some coefficients $\left(\gamma_{n}\right)_{n=1}^{\infty}$ in $\mathbb{C}$. The series $1 / S_{\mu}$ goes sometimes under the name of "T-transform of $\mu$ " (see [3]). We will use an explicit formula for how the coefficients of $R_{\mu}$ are expressed in terms of those of $1 / S_{\mu}$, which is presented in the next lemma. The proof of the lemma relies on a functional equation that is used in the study of the $R$-transform and is described as follows. Let $\left(s_{n}\right)_{n=1}^{\infty}$ and $\left(t_{n}\right)_{n=1}^{\infty}$ be two sequences of complex numbers such that the $s_{n}$ 's are expressed in terms of the $t_{n}$ 's by the formula

$$
s_{n}=\sum_{\substack{\pi=\left\{A_{1}, \ldots, A_{q}\right\} \\ \text { in } N C(n)}} t_{\left|A_{1}\right|} \cdots t_{\left|A_{q}\right|}, \quad \forall n \geq 1 .
$$

Then the series $f(z):=\sum_{n=1}^{\infty} t_{n} z^{n}$ and $g(z):=\sum_{n=1}^{\infty} s_{n} z^{n}$ satisfy the functional equation

$$
g(z)=f(z(1+g(z))) .
$$

Conversely, if the functional equation (6.7) is satisfied then it follows that the relations (6.6) hold. For the proof of these facts, see Theorem 16.15 in [11].

Lemma 6.2. Let $\mu$ be in $\mathcal{G}_{1}$, and consider the series $R_{\mu}, 1 / S_{\mu} \in \mathbb{C}[[z]]$, with coefficients denoted as in Equations (6.1) and (6.5), respectively. Then for every $n \geq 2$ we have that

$$
\alpha_{n}=\sum_{\substack{\pi=\left\{A_{1}, \ldots, A_{q}\right\} \\ \text { in } N C(n-1)}} \gamma_{\left|A_{1}\right|} \cdots \gamma_{\left|A_{q}\right|} .
$$

Proof. Consider the series

$$
g(z):=\sum_{n=1}^{\infty} \alpha_{n+1} z^{n}=\frac{1}{z} R_{\mu}(z)-1
$$


and

$$
f(z):=\sum_{n=1}^{\infty} \gamma_{n} z^{n}=1 / S_{\mu}(z)-1 .
$$

The required formula (6.8) says that the coefficients of $g$ are obtained from those of $f$ by summations over non-crossing partitions, exactly in the way described in Equation (6.6). Thus we may, equivalently, prove that $f$ and $g$ satisfy the functional equation (6.7).

We will obtain (6.7) by starting from the formula (6.4) which gives $S_{\mu}$ in terms of $R_{\mu}$. When explaining how this done, it is convenient to give a name to the identity series, say that we call it "id":

$$
\operatorname{id} \in \mathbb{C}[[z]], \quad \operatorname{id}(z)=z .
$$

Equation (6.4) says that id $\cdot S_{\mu}=R_{\mu}^{\langle-1\rangle}$, and this can be processed as follows:

$$
\begin{aligned}
\mathrm{id} \cdot S_{\mu}=R_{\mu}^{\langle-1\rangle} & \Rightarrow\left(\mathrm{id} \cdot S_{\mu}\right) \circ R_{\mu}=\mathrm{id} \\
& \Rightarrow\left(\mathrm{id} \circ R_{\mu}\right) \cdot\left(S_{\mu} \circ R_{\mu}\right)=\mathrm{id} \\
& \Rightarrow R_{\mu} \cdot\left(S_{\mu} \circ R_{\mu}\right)=\mathrm{id} \\
& \Rightarrow R_{\mu}=\mathrm{id} \cdot\left(1 /\left(S_{\mu} \circ R_{\mu}\right)\right) \\
& \left.\Rightarrow R_{\mu}=\mathrm{id} \cdot\left(\left(1 / S_{\mu}\right) \circ R_{\mu}\right)\right) .
\end{aligned}
$$

By using the latter equality and the relation between $R_{\mu}(z)$ and $g(z)$ we obtain that $g+1$ $=\left(1 / S_{\mu}\right) \circ R_{\mu}$, or equivalently, that

$$
g=\left(\left(1 / S_{\mu}\right)-1\right) \circ R_{\mu}
$$

But (6.11) implies (6.7), since $\left(1 / S_{\mu}\right)-1=f$ and $R_{\mu}(z)=z(1+g(z))$.

Remark 6.3. In this remark we collect a few basic facts about the algebra Sym of symmetric functions. We will deal with three of the commonly used sequences of generators for Sym:

$$
\left\{\begin{array}{l}
\text { the sequence }\left(e_{n}\right)_{n=1}^{\infty} \text { of elementary symmetric functions, } \\
\text { the sequence }\left(h_{n}\right)_{n=1}^{\infty} \text { of complete homogeneous symmetric functions, and } \\
\text { the sequence }\left(p_{n}\right)_{n=1}^{\infty} \text { of power sum symmetric functions. }
\end{array}\right.
$$

It is well-known that Sym can be identified to the commutative polynomial algebra generated by either of these three sequences (see Theorem 7.4.4 and Corollaries 7.6.2, 7.7.2 of [14, noting the slight difference that here we use $\mathbb{C}$ as field of scalars). Moreover, one has very nice formulas for passing from one sequence of generators to another, which are best expressed in terms of some suitably defined series in Sym $[[z]]$. For the present paper it is convenient to record these formulas as follows: consider the generating function for the $\left(e_{n}\right)_{n=1}^{\infty}$,

$$
\mathbf{e}(z):=1+\sum_{n=1}^{\infty} e_{n} z^{n}
$$

and the slightly modified generating functions for $\left(h_{n}\right)_{n=1}^{\infty}$ and $\left(p_{n}\right)_{n=1}^{\infty}$ defined by

$$
\mathbf{h}(z):=1+\sum_{n=1}^{\infty}(-1)^{n} h_{n} z^{n},
$$


and

$$
\mathbf{p}(z):=\sum_{n=1}^{\infty} \frac{(-1)^{n+1}}{n} p_{n} z^{n} .
$$

Then the formulas connecting the sequences $\left(e_{n}\right)_{n=1}^{\infty}$ and $\left(h_{n}\right)_{n=1}^{\infty}$ can be consolidated into the short statement that

$$
\mathbf{h}=1 / \mathbf{e} .
$$

Equation (6.15) is an equality holding in the algebra of series Sym $[[z]]$, where we use conventions of notation analogous to those presented for $\mathbb{C}[[z]]$ in Remark [6.1. Similarly, the formulas connecting $\left(e_{n}\right)_{n=1}^{\infty}$ and $\left(p_{n}\right)_{n=1}^{\infty}$ are consolidated into the equation

$$
\mathbf{p}=\log \mathbf{e}
$$

where $\log \mathbf{e} \in \operatorname{Sym}[[z]]$ is defined in the standard way (for series), by using the Taylor series of the logarithm:

$$
\log \mathbf{e}:=-\sum_{n=1}^{\infty} \frac{1}{n}(1-\mathbf{e})^{n} .
$$

For the proofs of these facts, we refer to Sections 7.6 and 7.7 of [14], or to pp. 12-16 in Chapter I.2 of [9].

Since in this paper we are dealing with the Hopf algebra structure of Sym, let us also record the formulas for the comultiplication of the three basic sequences of generators discussed above. For $\left(e_{n}\right)_{n=1}^{\infty}$ and $\left(h_{n}\right)_{n=1}^{\infty}$ one has that

$$
\left\{\begin{array}{l}
\Delta\left(e_{n}\right)=\sum_{i=0}^{n} e_{i} \otimes e_{n-i}, \quad \\
\Delta\left(h_{n}\right)=\sum_{i=0}^{n} h_{i} \otimes h_{n-i},
\end{array} \quad \forall n \geq 1\right.
$$

(where we make the convention that $e_{0}=h_{0}:=1$ ). On the other hand, every power sum symmetric function $p_{n}$ is a primitive element of Sym, in the sense that one has

$$
\Delta\left(p_{n}\right)=p_{n} \otimes 1+1 \otimes p_{n}, \quad \forall n \geq 1 .
$$

It is worth keeping in mind that conversely, every primitive homogeneous element of Sym must be a scalar multiple of some $p_{n}$ - see [18], Proposition 3.15 on p. 42 and Proposition 5.3 on p. 75 .

We now return to the space of distributions $\mathcal{G}_{1}$. As already mentioned in the introduction, we will use the $S$-transform in order to find a group isomorphism $\mu \mapsto \theta_{\mu}$ from $\left(\mathcal{G}_{1}, \otimes\right)$ onto the group $\mathbb{X}(\mathrm{Sym})$ of characters of Sym. The definition of $\theta_{\mu}$ is given next. For this definition recall that, since Sym can be viewed as the commutative polynomial algebra generated by $\left(h_{n}\right)_{n=1}^{\infty}$, a character of Sym can be defined (and is completely determined) by prescribing at will how we want it to act on the $h_{n}$ 's.

Definition 6.4. Let $\mu$ be a distribution in $\mathcal{G}_{1}$, and consider its $S$-transform,

$$
S_{\mu}(z)=: 1+\sum_{n=1}^{\infty} \beta_{n} z^{n} \in \mathbb{C}[[z]] .
$$

We denote by $\theta_{\mu}$ the character in $\mathbb{X}(\mathrm{Sym})$ which is uniquely determined by the requirement that

$$
\theta_{\mu}\left(h_{n}\right)=(-1)^{n} \beta_{n}, \quad \forall n \geq 1
$$


Proposition 6.5. The map $\mu \mapsto \theta_{\mu}$ is a group isomorphism from $\left(\mathcal{G}_{1}, \otimes\right)$ onto the group $\mathbb{X}($ Sym $)$ of characters of Sym, endowed with the operation of convolution.

Proof. It is immediate that the map $\mu \mapsto \theta_{\mu}$ is a bijection from $\mathcal{G}_{1}$ onto $\mathbb{X}(\mathrm{Sym})$ : it is injective because a distribution $\mu \in \mathcal{G}_{1}$ is completely determined by its $S$-transform, and it is surjective because every series in $\mathbb{C}[[z]]$ which has constant term equal to 1 appears as $S_{\mu}$ for some $\mu \in \mathcal{G}_{1}$. So the main point of the proof is to verify the group homomorphism property, i.e. that

$$
\theta_{\mu \bowtie \nu}=\theta_{\mu} \theta_{\nu}, \quad \forall \mu, \nu \in \mathcal{G}_{1} .
$$

We fix $\mu, \nu$ for which we will prove that (6.21) holds. Since both $\theta_{\mu \bowtie \nu}$ and $\theta_{\mu} \theta_{\nu}$ are in $\mathbb{X}(\mathrm{Sym})$, it will suffice to prove that they agree on the set of generators $\left(h_{n}\right)_{n=1}^{\infty}$ of Sym.

In view of how convolution of characters is defined, and by taking into account the formula (6.18) for the comultiplication of $h_{n}$, we see that:

$$
\left(\theta_{\mu} \theta_{\nu}\right)\left(h_{n}\right)=\sum_{i=0}^{n} \theta_{\mu}\left(h_{i}\right) \cdot \theta_{\nu}\left(h_{n-i}\right), \quad \forall n \geq 1 .
$$

Let us then consider the $S$-transforms of $\mu$ and of $\nu$, and let us write explicitly their coefficients:

$$
S_{\mu}(z)=: 1+\sum_{n=1}^{\infty} \beta_{n}^{\prime} z^{n}, \quad S_{\nu}(z)=: 1+\sum_{n=1}^{\infty} \beta_{n}^{\prime \prime} z^{n} .
$$

By invoking the definition of $\theta_{\mu}$ and $\theta_{\nu}$ on the right-hand side of (6.22) we find that for every $n \geq 1$ we have:

$$
\begin{aligned}
\left(\theta_{\mu} \theta_{\nu}\right)\left(h_{n}\right) & =\sum_{i=0}^{n}\left((-1)^{i} \beta_{i}^{\prime}\right) \cdot\left((-1)^{n-i} \beta_{n-i}^{\prime \prime}\right) \\
& =(-1)^{n} \cdot\left(\text { coeff. of } z^{n} \text { in } S_{\mu} S_{\nu}\right) \\
& =(-1)^{n} \cdot\left(\text { coeff. of } z^{n} \text { in } S_{\mu 凶 \nu}\right) ;
\end{aligned}
$$

at the last equality sign we used the multiplicativity property of the $S$-transform, which says precisely that $S_{\mu 凶 \nu}=S_{\mu} \cdot S_{\nu}$. Clearly, the above sequence of equalities has ended with $\theta_{\mu \bowtie \nu}\left(h_{n}\right)$, and this concludes the proof.

Remark 6.6. The reader may have noticed that the proof of Proposition 6.5 would work fine (and would look simpler) without the sign " $(-1)^{n}$ " in front of $h_{n}$. Also, with or without signs, the proof would work equally well with $e_{n}$ 's instead of the $h_{n}$ 's, since the $e_{n}$ 's comultiply in exactly the same way as the $h_{n}$ 's in Equation (6.18).

Hence there were in fact several possible alternatives for the definition given above to $\theta_{\mu}$. The justification for the choice selected in Definition 6.4 is that it gives an underlying homomorphism $\mathcal{Y}^{(1)} \rightarrow$ Sym which matches the "combinatorial Hopf algebra" structure from [1]. This is discussed in more detail in Remark 7.7 and Proposition 7.8 below. Right now let us only insist on the point that it does indeed make sense to look for an "underlying homomorphism" in connection to the definition of $\theta_{\mu}$; this is because we have found $\left(\mathcal{G}_{1}, \otimes\right)$ to be isomorphic both to $\mathbb{X}\left(\mathcal{Y}^{(1)}\right)$ (via $\mu \mapsto \chi_{\mu}$ ) and to $\mathbb{X}($ Sym $)\left(\right.$ via $\mu \mapsto \theta_{\mu}$ ), and it is natural to try to connect these two isomorphisms via a homomorphism $\Phi: \mathcal{Y}^{(1)} \rightarrow$ Sym. The homomorphism $\Phi$ will be introduced in Definition 6.8 below. Before introducing it, we would like to make some comments about $\theta_{\mu}$. 
Remark 6.7. For $\mu \in \mathcal{G}_{1}$ one has a natural way of extending the character $\theta_{\mu}:$ Sym $\rightarrow \mathbb{C}$ to a unital homomorphism $\Theta_{\mu}: \operatorname{Sym}[[z]] \rightarrow \mathbb{C}[[z]]$, where a series $\mathbf{u}(z)=\sum_{n=0}^{\infty} u_{n} z^{n} \in$ Sym $[[z]]$ is mapped by $\Theta_{\mu}$ to the series $\sum_{n=0}^{\infty} \theta_{\mu}\left(u_{n}\right) z^{n} \in \mathbb{C}[[z]]$. It is useful to record what $\Theta_{\mu}$ does to the special series $\mathbf{e}, \mathbf{h}, \mathbf{p} \in \operatorname{Sym}[[z]]$ from Equations (6.12)-(6.14) of Remark 6.3. First of all, from the very definition of $\Theta_{\mu}$ it is clear that

$$
\Theta_{\mu}(\mathbf{h})=S_{\mu} .
$$

Then from Equation (6.15) written in the form $\mathbf{e}=1 / \mathbf{h}$ and the fact that $\Theta_{\mu}$ is a unital homomorphism, we infer that

$$
\Theta_{\mu}(\mathbf{e})=1 / S_{\mu} .
$$

Finally, let us observe that when taking the logarithm of the left-hand side of (6.24), the log may be as well written inside the brackets; this is because $\log \left(\Theta_{\mu}(\mathbf{e})\right)$ and $\Theta_{\mu}(\log \mathbf{e})$ are both defined by using the Taylor series of the logarithm, and because of the homomorphic properties of $\Theta_{\mu}$. When we also invoke Equation (6.16), we thus find that

$$
\Theta_{\mu}(\mathbf{p})=\Theta_{\mu}(\log \mathbf{e})=\log \left(\Theta_{\mu}(\mathbf{e})\right)=\log \left(1 / S_{\mu}\right)=-\log S_{\mu} .
$$

Definition 6.8. $1^{o}$ For every $n \geq 2$ we denote

$$
y_{n}=\sum_{\substack{\pi=\left\{A_{1}, \ldots, A_{q}\right\} \\ \text { in } N C(n-1)}} e_{\left|A_{1}\right|} \cdots e_{\left|A_{q}\right|} \in \text { Sym. }
$$

Thus for instance for $2 \leq n \leq 5$ we have

$$
\left\{\begin{array}{l}
y_{2}=e_{1}, y_{3}=e_{2}+e_{1}^{2}, y_{4}=e_{3}+3 e_{1} e_{2}+e_{1}^{3}, \\
y_{5}=e_{4}+4 e_{1} e_{3}+2 e_{2}^{2}+6 e_{1}^{2} e_{2}+e_{1}^{4} .
\end{array}\right.
$$

Clearly, every $y_{n}$ is a homogeneous symmetric function of degree $n-1$.

$2^{o}$ We denote by $\Phi$ the unital algebra homomorphism from $\mathcal{Y}^{(1)}\left(=\mathbb{C}\left[Y_{2}, Y_{3}, \ldots\right]\right)$ to Sym which is uniquely determined by the requirement that $\Phi\left(Y_{n}\right)=y_{n}, \forall n \geq 2$.

Proposition 6.9. For every $\mu \in \mathcal{G}_{1}$ we have that $\chi_{\mu}=\theta_{\mu} \circ \Phi$, where $\chi_{\mu} \in \mathbb{X}\left(\mathcal{Y}^{(1)}\right)$ and $\theta_{\mu} \in \mathbb{X}($ Sym $)$ are as in Definition 1.1 and Definition 6.4, respectively.

Proof. Both $\chi_{\mu}$ and $\theta_{\mu} \circ \Phi$ are characters of $\mathcal{Y}^{(1)}$, so in order to show that they are equal to each other it suffices to verify that they agree on every $Y_{n}, n \geq 2$.

Let us consider the $R$-transform of $\mu$,

$$
R_{\mu}(z)=z+\sum_{n=2}^{\infty} \alpha_{n} z^{n}
$$

and let us recall that $\chi_{\mu}$ is defined via the requirement that $\chi_{\mu}\left(Y_{n}\right)=\alpha_{n}, \forall n \geq 2$. Hence what we have to show is that $\left(\theta_{\mu} \circ \Phi\right)\left(Y_{n}\right)=\alpha_{n}$, or equivalently, that

$$
\theta_{\mu}\left(y_{n}\right)=\alpha_{n}, \quad \forall n \geq 2 \text {. }
$$

On the other hand let us also consider the series

$$
1 / S_{\mu}(z)=: 1+\sum_{n=1}^{\infty} \gamma_{n} z^{n}
$$


In Remark 6.7 it was observed that $\Theta_{\mu}(\mathbf{e})=1 / S_{\mu}$; when we write this equation in coefficients, it says that $\theta_{\mu}\left(e_{n}\right)=\gamma_{n}, \forall n \geq 1$. Consequently, when we apply the linear and multiplicative functional $\theta_{\mu}$ to both sides of Equation (6.26) we find that

$$
\theta_{\mu}\left(y_{n}\right)=\sum_{\substack{\pi=\left\{A_{1}, \ldots, A_{q}\right\} \\ \text { in } N C(n-1)}} \gamma_{\left|A_{1}\right|} \cdots \gamma_{\left|A_{q}\right|}
$$

and (6.28) follows from Lemma 6.2 .

Based on Proposition 6.9, we can now place the one-dimensional instance of the $L S$ transform in the framework of Sym.

Proposition 6.10. For a distribution $\mu \in \mathcal{G}_{1}$, the $L S$-transform $L S_{\mu}$ can be written in the form

$$
L S_{\mu}(z)=\sum_{n=2}^{\infty}\left(\left(\log \theta_{\mu}\right)\left(y_{n}\right)\right) z^{n}
$$

where $\theta_{\mu} \in \mathbb{X}($ Sym $)$ is as in Definition 6.4.

Proof. As pointed out in Equation (1.15) of the introduction, in the one-dimensional case the definition of the $L S$-transform simplifies to

$$
L S_{\mu}(z)=\sum_{n=2}^{\infty}\left(\left(\log \chi_{\mu}\right)\left(Y_{n}\right)\right) z^{n} .
$$

Hence proving (6.29) amounts to verifying that $\left(\log \theta_{\mu}\right)\left(y_{n}\right)=\left(\log \chi_{\mu}\right)\left(Y_{n}\right), \forall n \geq 2$. Clearly, this will follow if we can prove that $\left(\log \theta_{\mu}\right) \circ \Phi=\log \chi_{\mu}$, where $\Phi: \mathcal{Y}^{(1)} \rightarrow$ Sym is as in Definition 6.8.2. Since Proposition 6.9 gives us that $\theta_{\mu} \circ \Phi=\chi_{\mu}$, it will thus suffice to verify that for every character $\xi \in \mathbb{X}(\mathrm{Sym})$ we have

$$
(\log \xi) \circ \Phi=\log (\xi \circ \Phi)
$$

(equality of linear functionals on $\mathcal{Y}^{(1)}$ ).

In order to prove (6.30), we start from the following fact:

$$
\left\{\begin{array}{c}
\mathbb{X}(\mathrm{Sym}) \ni \xi \mapsto \xi \circ \Phi \in \mathbb{X}\left(\mathcal{Y}^{(1)}\right) \\
\text { is a group homomorphism }
\end{array}\right\}
$$

This holds because, as shown in Proposition 6.9, the map in (6.31) is the one which connects the two group isomorphisms $\mu \mapsto \chi_{\mu}$ and $\mu \mapsto \theta_{\mu}$ going from $\mathcal{G}_{1}$ to $\mathbb{X}\left(\mathcal{Y}^{(1)}\right)$ and to $\mathbb{X}(\operatorname{Sym})$, respectively. 3 From (6.31) we infer that for every $\xi \in \mathbb{X}($ Sym $)$ and every $\ell \geq 1$ we have

$$
\left(\varepsilon_{\mathrm{Sym}}-\xi\right)^{\ell} \circ \Phi=\left(\varepsilon_{\mathcal{Y}^{(1)}}-(\xi \circ \Phi)\right)^{\ell}
$$

\footnotetext{
${ }^{3}$ We emphasize that at this point we are not using any properties that $\Phi$ might have in connection to the coalgebra structures of $\mathcal{Y}^{(1)}$ and Sym; for the time being $\Phi$ is merely a unital algebra homomorphism.
} 
(equality of functionals on $\mathcal{Y}^{(1)}$, where $\varepsilon_{\text {Sym }}$ and $\varepsilon_{\mathcal{Y}^{(1)}}$ are counits, and the convolution powers are taken in the suitable algebras of functionals). Indeed, we have

$$
\begin{aligned}
\left(\varepsilon_{\mathrm{Sym}}-\xi\right)^{\ell} \circ \Phi & =\left(\sum_{j=0}^{\ell}\left(\begin{array}{l}
\ell \\
j
\end{array}\right)(-1)^{j} \xi^{j}\right) \circ \Phi \\
& =\sum_{j=0}^{\ell}\left(\begin{array}{l}
\ell \\
j
\end{array}\right)(-1)^{j}\left(\xi^{j} \circ \Phi\right) \\
& =\sum_{j=0}^{\ell}\left(\begin{array}{l}
\ell \\
j
\end{array}\right)(-1)^{j}(\xi \circ \Phi)^{j} \quad(\text { by (6.31) }) \\
& =\left(\varepsilon_{\mathcal{Y}^{(1)}}-(\xi \circ \Phi)\right)^{\ell} .
\end{aligned}
$$

Finally we are just left to observe that for every $\xi \in \mathbb{X}(\mathrm{Sym})$ we have

$$
\begin{aligned}
(\log \xi) \circ \Phi & =\left(-\sum_{\ell=1}^{\infty} \frac{1}{\ell}\left(\varepsilon_{\mathrm{Sym}}-\xi\right)^{\ell}\right) \circ \Phi \\
& =-\sum_{\ell=1}^{\infty} \frac{1}{\ell}\left(\varepsilon_{\mathcal{Y}^{(1)}}-(\xi \circ \Phi)\right)^{\ell} \quad(\text { by }(\underline{6.32})) \\
& =\log (\xi \circ \Phi),
\end{aligned}
$$

as claimed in (6.30).

It is now easy to obtain the precise formula relating the $L S$-transform with the log of the $S$-transform - we will only have to combine Proposition 6.10 with some basic properties of the infinitesimal characters of Sym, which are recorded in the next lemma.

Lemma 6.11. $1^{o}$ Let $\xi$ be an infinitesimal character in $\mathbb{I}($ Sym $)$, and let $u_{1}, u_{2}$ be homogeneous symmetric functions of degrees $\geq 1$. Then $\xi\left(u_{1} u_{2}\right)=0$.

$2^{o}$ Let $\eta$ be a character in $\mathbb{X}($ Sym $)$, and consider its logarithm $\xi:=\log \eta \in \mathbb{I}($ Sym $)$. Then

$$
\xi\left(p_{n}\right)=\eta\left(p_{n}\right), \quad \forall n \geq 1 .
$$

$3^{o}$ Let $\xi$ be an infinitesimal character in $\mathbb{I}($ Sym $)$. Then we have that

$$
\xi\left(y_{n}\right)=\frac{(-1)^{n}}{n-1} \xi\left(p_{n-1}\right), \quad \forall n \geq 2 .
$$

Proof. $1^{\circ}$ We have that

$$
\begin{aligned}
\xi\left(u_{1} u_{2}\right) & \left.=\xi\left(u_{1}\right) \varepsilon\left(u_{2}\right)+\varepsilon\left(u_{1}\right) \xi\left(u_{2}\right) \quad \text { (by the definition of } \mathbb{I}(\mathrm{Sym})\right) \\
& =\xi\left(u_{1}\right) \cdot 0+0 \cdot \xi\left(u_{2}\right) \quad(\text { by the definition of } \varepsilon) \\
& =0 .
\end{aligned}
$$

$2^{o}$ Note that $(\varepsilon-\eta)(1)=1-1=0$, which implies that for every $\ell \geq 1$ we have

$$
(\varepsilon-\eta)^{\ell}(1)=((\varepsilon-\eta) \otimes \cdots \otimes(\varepsilon-\eta))(1 \otimes \cdots \otimes 1)=0 .
$$

For $\ell \geq 2$ this implies that

$$
(\varepsilon-\eta)^{\ell}\left(p_{n}\right)=\left((\varepsilon-\eta)^{\ell-1} \otimes(\varepsilon-\eta)\right)\left(p_{n} \otimes 1+1 \otimes p_{n}\right)=0
$$


(where we wrote $(\varepsilon-\eta)^{\ell}=(\varepsilon-\eta)^{\ell-1} \cdot(\varepsilon-\eta)$, and we used formula (6.19) for $\Delta\left(p_{n}\right)$ ). Hence indeed, we find that

$$
\xi\left(p_{n}\right)=-\sum_{\ell=1}^{\infty} \frac{1}{\ell}\left((\varepsilon-\eta)^{\ell}\right)\left(p_{n}\right)=-(\varepsilon-\eta)\left(p_{n}\right)=\eta\left(p_{n}\right) .
$$

$3^{o}$ Equation (6.26) which defines $y_{n}$ can also be written in the form

$$
y_{n}=e_{n-1}+\sum_{\substack{\pi=\left\{A_{1}, \ldots, A_{q}\right\} \\ \text { in } N C(n-1), q \geq 2}} e_{\left|A_{1}\right|} \cdots e_{\left|A_{q}\right|} ;
$$

hence by using part $1^{\circ}$ of the lemma we find that

$$
\xi\left(y_{n}\right)=\xi\left(e_{n-1}\right), \quad \forall n \geq 2 .
$$

Now let us look again at Equation (6.16) which connects the series $\mathbf{e}, \mathbf{p} \in \operatorname{Sym}[[z]]$. By taking derivative with respect to $z$ we obtain that $\mathbf{p}^{\prime} \cdot \mathbf{e}=\mathbf{e}^{\prime}$, and when written coefficientwise this says that

$$
p_{1} e_{n-1}-p_{2} e_{n-2}+\cdots+(-1)^{n} p_{n-1} e_{1}+(-1)^{n+1} p_{n}=n e_{n}, \quad \forall n \geq 1 .
$$

We apply $\xi$ to both sides of (6.36) and invoke part $1^{\circ}$ of the lemma to conclude that

$$
(-1)^{n+1} \xi\left(p_{n}\right)=n \xi\left(e_{n}\right), \quad \forall n \geq 1 .
$$

Clearly, the required Equation (6.34) follows from (6.35) and (6.37).

Corollary 6.12. Let $\mu$ be a distribution in $\mathcal{G}_{1}$, and consider the power series $S_{\mu}, L S_{\mu} \in$ $\mathbb{C}[[z]]$. Then $L S_{\mu}(z)=-z \log S_{\mu}(z)$.

Proof. The constant and the linear term vanish in both $L S_{\mu}(z)$ and $-z \log S_{\mu}(z)$, hence we only have to look at coefficients of $z^{n}$ for $n \geq 2$.

So let us fix an integer $n \geq 2$. By Proposition 6.10, the coefficient of $z^{n}$ in $L S_{\mu}$ is equal to $\left(\log \theta_{\mu}\right)\left(y_{n}\right)$. But on the other hand we have that

$$
\begin{aligned}
\left(\log \theta_{\mu}\right)\left(y_{n}\right) & =\frac{(-1)^{n}}{n-1}\left(\log \theta_{\mu}\right)\left(p_{n-1}\right)(\text { by Lemma 6.11, } 3) \\
& =\frac{(-1)^{n}}{n-1} \theta_{\mu}\left(p_{n-1}\right)(\text { by Lemma 6.11, } 2) \\
& \left.=\theta_{\mu}\left(\text { coefficient of } z^{n-1} \text { in } \mathbf{p}\right)(\text { by } \underline{6.14})\right) \\
& =\text { coefficient of } z^{n-1} \text { in } \Theta_{\mu}(\mathbf{p}) \\
& =\text { coefficient of } z^{n-1} \text { in }\left(-\log S_{\mu}\right)
\end{aligned}
$$

where at the last two equality signs we used the homomorphism $\Theta_{\mu}$ from Remark 6.7, and its property observed in Equation (6.25). So we obtained equality with the coefficient of $z^{n}$ in $-z \log S_{\mu}(z)$, as required. 


\section{Some remarks about $\mathcal{Y}^{(1)}$}

The goal of the present section is to clarify a few points concerning the Hopf algebra $\mathcal{Y}^{(1)}$, that arise naturally from the considerations in Section 6 .

We will continue to use the same framework and notations as in Section 6. Recall that the connection between $\mathcal{Y}^{(1)}$ and symmetric functions was formally achieved by the homomorphism $\Phi: \mathcal{Y}^{(1)} \rightarrow$ Sym from Definition 6.8,2. In Section 6 it came in handy to introduce and use $\Phi$ only in its capacity of homomorphism of unital algebras; but $\Phi$ is in fact more than that - it is an isomorphism of graded Hopf algebras. This will be proved in Theorem 7.5 below. Our method of proof will be to observe that $\mathcal{Y}^{(1)}$ is cocommutative, and then to take advantage of the special properties that primitive elements are known to have in cocommutative Hopf algebras.

Lemma 7.1. The Hopf algebra $\mathcal{Y}^{(1)}$ is cocommutative.

Proof. We have to show that $F \circ \Delta=\Delta$, where $F: \mathcal{Y}^{(1)} \otimes \mathcal{Y}^{(1)} \rightarrow \mathcal{Y}^{(1)} \otimes \mathcal{Y}^{(1)}$ is the flip automorphism acting by $F(P \otimes Q)=Q \otimes P$ for $P, Q \in \mathcal{Y}^{(1)}$. Since both $\Delta$ and $F \circ \Delta$ are unital algebra homomorphisms from $\mathcal{Y}^{(1)}$ to $\mathcal{Y}^{(1)} \otimes \mathcal{Y}^{(1)}$, it suffices to verify that they agree on the set of generators $\left\{Y_{n} \mid n \geq 2\right\}$ of $\mathcal{Y}^{(1)}$.

So let us fix an $n \geq 2$. The formula defining $\Delta$ (see Equation (3.2) in Definition [3.2.1) gives us here that

$$
\Delta\left(Y_{n}\right)=\sum_{\pi \in N C(n)} Y_{\pi} \otimes Y_{K(\pi)}
$$

where for $\pi=\left\{A_{1}, \ldots, A_{q}\right\} \in N C(n)$ we put

$$
Y_{\pi}:=Y_{\left|A_{1}\right|} \cdots Y_{\left|A_{q}\right|} \cdot
$$

On the other hand we have

$$
\begin{aligned}
(F \circ \Delta)\left(Y_{n}\right) & =F\left(\sum_{\pi \in N C(n)} Y_{\pi} \otimes Y_{K(\pi)}\right) \\
& =\sum_{\pi \in N C(n)} Y_{K(\pi)} \otimes Y_{\pi} \\
& \left.=\sum_{\rho \in N C(n)} Y_{\rho} \otimes Y_{K^{-1}(\rho)} \quad \text { (by substitution } K(\pi)=\rho\right) .
\end{aligned}
$$

Now, it is immediately seen that for every $\rho \in N C(n)$, the partitions $K(\rho)$ and $K^{-1}(\rho)$ have the same block structure - that is, they can be written as

$$
\left\{\begin{array}{c}
K(\rho)=\left\{B_{1}, \ldots, B_{q}\right\}, \quad K^{-1}(\rho)=\left\{C_{1}, \ldots, C_{q}\right\} \\
\text { with }\left|B_{1}\right|=\left|C_{1}\right|, \ldots,\left|B_{q}\right|=\left|C_{q}\right| .
\end{array}\right.
$$

(See Exercise 9.23 on page 147 of [11]; or directly from formula (2.1) in Notation [2.1, observe that the associated permutations $P_{K(\rho)}=P_{\rho}^{-1} P_{1_{n}}$ and $P_{K^{-1}(\rho)}=P_{1_{n}} P_{\rho}^{-1}$ belong to the same conjugacy class of the symmetric group.) From (17.3) it is immediate that $Y_{K^{-1}(\rho)}=Y_{K(\rho)}, \forall \rho \in N C(n)$, so we find that

$$
(F \circ \Delta)\left(Y_{n}\right)=\sum_{\rho \in N C(n)} Y_{\rho} \otimes Y_{K(\rho)}=\Delta\left(Y_{n}\right)
$$

as required. 
Remark 7.2. Consider the set of primitive elements of $\mathcal{Y}^{(1)}$,

$$
\operatorname{Prim}\left(\mathcal{Y}^{(1)}\right):=\left\{P \in \mathcal{Y}^{(1)} \mid \Delta(P)=P \otimes 1+1 \otimes P\right\} .
$$

The commutator operation $[P, Q]:=P Q-Q P$ turns $\operatorname{Prim}\left(\mathcal{Y}^{(1)}\right)$ into a Lie algebra, and a fundamental result from the theory of cocommutative Hopf algebras (the Cartier-KostantMilnor-Moore theorem, see e.g. Theorem 13.0.1 on p. 274 of [15]) allows us to identify $\mathcal{Y}^{(1)}$ as the universal envelopping algebra of $\operatorname{Prim}\left(\mathcal{Y}^{(1)}\right)$. For our discussion here, geared towards the proof of Theorem 7.5 below, it will suffice to use the weaker statement that $\operatorname{Prim}\left(\mathcal{Y}^{(1)}\right)$ generates $\mathcal{Y}^{(1)}$ as a unital algebra - see Corollary 13.0.3 on p. 278 of [15]. Since it is immediate that every primitive element can be written as a sum of homogeneous primitive elements, the statement about primitives that we want to retain can thus be recorded as follows:

$$
\left\{\begin{array}{c}
\mathcal{Y}^{(1)} \text { is generated as a unital algebra } \\
\text { by the set of its homogeneous primitive elements }
\end{array}\right\} \text {. }
$$

Before proceeding to the Theorem 7.5 we will also record, in Lemma 7.4, a simple observation about homogeneous symmetric functions.

Notation 7.3. Let $n$ be a positive integer.

$1^{o}$ We use the standard notation " $\lambda \vdash n$ " to denote a partition $\lambda$ of the number $n$ (that is, $\lambda$ is a tuple $\left(i_{1}, i_{2}, \ldots, i_{m}\right)$ of integers $i_{1} \geq i_{2} \geq \cdots \geq i_{m}>0$ with $\left.i_{1}+i_{2}+\cdots+i_{m}=n\right)$. For $\lambda=\left(i_{1}, \ldots, i_{m}\right) \vdash n$, the number $m$ of parts of $\lambda$ is called the length of $\lambda$, and is denoted by $\ell(\lambda)$.

$2^{o}$ We denote by $\operatorname{Sym}_{n}$ the vector space of homogeneous symmetric functions of degree $n$. In $\operatorname{Sym}_{n}$ we will use the linear basis $\left\{p_{\lambda} \mid \lambda \vdash n\right\}$, where for every $\lambda=\left(i_{1}, i_{2}, \ldots, i_{m}\right) \vdash n$ we put

$$
p_{\lambda}:=p_{i_{1}} p_{i_{2}} \cdots p_{i_{m}} \in \operatorname{Sym}_{n} .
$$

(For the verification that $\left\{p_{\lambda} \mid \lambda \vdash n\right\}$ is indeed a basis for $\operatorname{Sym}_{n}$, see e.g. Corollary 7.7.2 on p. 298 of [14].)

Lemma 7.4. Let $n$ be a positive integer and let $u$ be a symmetric function in Sym $_{n}$ which has the property that

$$
\xi^{2}(u)=2 \xi(u), \quad \forall \xi \in \mathbb{X}(S y m)
$$

Then $u \in \mathbb{C} p_{n}$.

Proof. Consider the writing of $u$ in terms of the basis $\left\{p_{\lambda} \mid \lambda \vdash n\right\}$ :

$$
u=\sum_{\lambda \vdash n} t_{\lambda} p_{\lambda}, \quad \text { with }\left(t_{\lambda}\right)_{\lambda \vdash n} \text { from } \mathbb{C} .
$$

We leave it as an exercise to the reader to use the formula defining $\xi^{2}$ and the fact that each of $p_{1}, p_{2}, \ldots, p_{n}$ is a primitive element of Sym in order to verify that (17.8) implies:

$$
\xi^{2}(u)=2 \xi(u)+\sum_{\substack{\lambda \vdash n, \ell(\lambda) \geq 2}} t_{\lambda} N(\lambda) \xi\left(p_{\lambda}\right), \quad \forall \xi \in \mathbb{X}(\mathrm{Sym}),
$$

where for every $\lambda \vdash n$ with $\ell(\lambda) \geq 2, N(\lambda)$ is a positive integer depending only on $\lambda$. (The combinatorial significance of $N(\lambda)$ is that it counts in how many ways $\lambda$ can be obtained 
by merging together two partitions $\lambda^{\prime} \vdash m$ and $\lambda^{\prime \prime} \vdash n-m$ for some $1 \leq m<n$.) The hypothesis given on $u$ thus amounts to the fact that

$$
\sum_{\substack{\lambda \vdash n, \ell(\lambda) \geq 2}} t_{\lambda} N(\lambda) \xi\left(p_{\lambda}\right)=0, \quad \forall \xi \in \mathbb{X}(\mathrm{Sym}) .
$$

But a character $\xi \in \mathbb{X}(\mathrm{Sym})$ may take any prescribed set of values on $p_{1}, p_{2}, \ldots, p_{n}$; by using this fact it is easy to see that (17.10) can only hold if $t_{\lambda} N(\lambda)=0$ (hence $t_{\lambda}=0$ ) for every $\lambda \vdash n$ with $\ell(\lambda) \geq 2$, and the conclusion that $u \in \mathbb{C} p_{n}$ follows.

Theorem 7.5. The map $\Phi: \mathcal{Y}^{(1)} \rightarrow$ Sym from Definition 6.8 . 2 is an isomorphism of graded Hopf algebras.

Proof. $\Phi$ is by definition a unital algebra homomorphism. Directly from the definition it is also immediate that $\Phi$ respects the gradings considered on $\mathcal{Y}^{(1)}$ and on Sym, and that $\Phi$ respects counits (we have $\varepsilon_{\mathrm{Sym}} \circ \Phi=\varepsilon_{\mathcal{Y}^{(1)}}$ ).

We next verify that $\Phi$ is surjective. It clearly suffices to verify that $\Phi\left(\mathcal{Y}^{(1)}\right) \ni e_{n}$ for every $n \geq 1$, which we do by induction on $n$. The base case $n=1$ holds because $e_{1}=y_{2}=\Phi\left(Y_{2}\right)$. For the induction step let us assume that $e_{1}, \ldots, e_{n-1}$ belong to $\Phi\left(\mathcal{Y}^{(1)}\right)$, and let us prove that $e_{n}$ belongs to $\Phi\left(\mathcal{Y}^{(1)}\right)$ as well, for some $n \geq 2$. To this end we observe that the formula used to define $y_{n+1}$ in Definition 6.8,1 can be written in the form

$$
e_{n}=y_{n+1}-\sum_{\substack{\pi=\left\{A_{1}, \ldots, A_{q}\right\} \text { in } \\ N C(n), \text { with } q \geq 2}} e_{\left|A_{1}\right|} \cdots e_{\left|A_{q}\right|} .
$$

The right-hand side of (7.11) is a polynomial expression in $y_{n+1}$ and $e_{1}, \ldots, e_{n-1}$, which all belong to $\Phi\left(\mathcal{Y}^{(1)}\right)$. Since $\Phi\left(\mathcal{Y}^{(1)}\right)$ is a subalgebra of Sym, we thus conclude that $e_{n} \in \Phi\left(\mathcal{Y}^{(1)}\right)$, as required.

Now, in the context where we know that $\Phi$ is surjective and respects the gradings, the injectivity of $\Phi$ will also follow if we can check that for every $n \geq 0$ the homogeneous spaces $\mathcal{Y}_{n}^{(1)} \subseteq \mathcal{Y}^{(1)}$ and $\operatorname{Sym}_{n} \subseteq$ Sym have the same finite dimension. And indeed, $\operatorname{dim}\left(\mathcal{Y}_{0}^{(1)}\right)=$ $\operatorname{dim}\left(\operatorname{Sym}_{0}\right)=1$, while for every $n \geq 1$ we have

$$
\operatorname{dim}\left(\operatorname{Sym}_{n}\right)=|\{\lambda \mid \lambda \vdash n\}|=\operatorname{dim}\left(\mathcal{Y}_{n}^{(1)}\right) .
$$

The first of the two equalities in (7.12) follows from the fact that $\left\{p_{\lambda} \mid \lambda \vdash n\right\}$ is a basis for $\operatorname{Sym}_{n}$, while the second is an immediate consequence of the fact that $\mathcal{Y}^{(1)}$ is just $\mathbb{C}\left[Y_{2}, Y_{3}, \ldots\right]$, with degree $\left(Y_{m}\right)=m-1$ for every $m \geq 2$. This completes the verifications that $\Phi$ is bijective.

We are left to prove that $\Phi$ respects the comultiplications of $\mathcal{Y}^{(1)}$ and Sym, i.e. that

$$
\Delta_{\mathrm{Sym}} \circ \Phi=(\Phi \otimes \Phi) \circ \Delta_{\mathcal{Y}^{(1)}} .
$$

Due to (7.5) of Remark 7.2 and the fact that both sides of Equation (7.13) are unital homomorphisms from $\mathcal{Y}^{(1)}$ to $\mathrm{Sym} \otimes \mathrm{Sym}$, it is sufficient to verify that

$$
\left(\Delta_{\mathrm{Sym}} \circ \Phi\right)(P)=\left((\Phi \otimes \Phi) \circ \Delta_{\mathcal{Y}^{(1)}}\right)(P)
$$


when $P$ is a homogeneous primitive element of $\mathcal{Y}^{(1)}$. So let $P$ be a such an element, of degree $n$. We claim that the symmetric function $u:=\Phi(P) \in \operatorname{Sym}_{n}$ satisfies the hypothesis of Lemma 7.4. Indeed, for every character $\xi \in \mathbb{X}(\mathrm{Sym})$ we write:

$$
\begin{aligned}
\xi^{2}(u) & =\left(\xi^{2} \circ \Phi\right)(P) \\
& =(\xi \circ \Phi)^{2}(P) \quad(\text { by }(6.31) \text { in proof of Proposition 6.10) } \\
& =((\xi \circ \Phi) \otimes(\xi \circ \Phi))(P \otimes 1+1 \otimes P) \\
& =2 \xi(u) .
\end{aligned}
$$

Hence Lemma 7.4 applies and gives us that $u$ is a scalar multiple of the power sum symmetric function $p_{n}$. This implies in particular that $u$ is primitive in Sym, and it follows that

$$
\left(\Delta_{\mathrm{Sym}} \circ \Phi\right)(P)=u \otimes 1+1 \otimes u=\left((\Phi \otimes \Phi) \circ \Delta_{\mathcal{Y}^{(1)}}\right)(P),
$$

as we wanted.

Remark 7.6. In the proof of Theorem [7.5, an alternative approach to the fact that $\Phi$ respects comultiplication would go by proving, directly from the definitions, that every symmetric function $y_{n}$ comultiplies in Sym by the same formula as the one used for the comultiplication of $Y_{n} \in \mathcal{Y}^{(1)}$. That is, one could go for a direct proof of the formula:

$$
\Delta\left(y_{n}\right)=\sum_{\pi \in N C(n)} y_{\pi} \otimes y_{K(\pi)}, \quad \forall n \geq 2,
$$

where for $\pi=\left\{A_{1}, A_{2}, \ldots, A_{q}\right\} \in N C(n)$ we put

$$
y_{\pi}:=y_{\left|A_{1}\right|} y_{\left|A_{2}\right|} \cdots y_{\left|A_{q}\right|} \in \mathrm{Sym}
$$

with the convention that $y_{1}:=1$. By "direct proof" we mean here a direct derivation of (7.14) from Equation (6.26) which defines the $y_{n}$ 's in terms of $e_{n}$ 's, combined with the simple formula (6.18) for the comultiplication of $e_{n}$. (It is instructive to see how this works for some concrete small values of $n$, e.g. pick $n=3$ and verify directly that $\Delta\left(y_{3}\right)=$ $y_{3} \otimes 1+3 y_{2} \otimes y_{2}+1 \otimes y_{3}$.)

For the readers who want to try their hand at the direct derivation of (7.14), we offer the following tip: the formula defining $y_{n}$ in (6.26) can be re-written in the form of a recursion,

$$
y_{n}=\sum_{m=2}^{n}\left(e_{m-1} \cdot \sum_{1=i_{1}<i_{2}<\cdots<i_{m}=n} y_{i_{2}-i_{1}} y_{i_{3}-i_{2}} \cdots y_{i_{m}-i_{m-1}}\right) .
$$

This recursion is essentially equivalent to the "functional equation of the $R$-transform" from Lecture 16 of [11, which was also invoked in the proof of Lemma 6.2. One can use (7.16) and the formula (6.18) for the comultiplication of the $e_{n}$ 's in order to prove by induction on $n$ that (7.14) holds. Proceeding by induction somewhat simplifies the argument, but this "direct proof" that $\Phi$ respects comultiplication still remains (by quite a bit) more involved than the approach which we chose to present in the proof shown above for Theorem 7.5.

Another way of looking at the homomorphism $\Phi: \mathcal{Y}^{(1)} \rightarrow$ Sym is by placing it in the framework of combinatorial Hopf algebras from [1. We will explain how this goes in the next remark, where it is convenient to start by pointing out a connection which $\mathcal{Y}^{(1)}$ has with the theory of incidence Hopf algebras. 
Remark 7.7. $1^{o}$ Let $\mathcal{R}$ be Rota's Hopf algebra of isomorphism classes of finite graded posets. We use the name "Rota's Hopf algebra" and the notation $\mathcal{R}$ by following Example 2.2 of [1]. Standard references for this graded connected Hopf algebra are [7, [12. Here is a brief summary of facts about $\mathcal{R}$ that we want to refer to. In (i)-(iv) below, by "finite graded poset" we mean a finite poset $G$ with minimum element $0_{G}$ and maximum element $1_{G}$, such that all the saturated chains in $G$ have the same length $r$; the number $r$ is called the rank of the poset.

(i) For every finite graded poset $G$ one has a homogeneous element $\bar{G} \in \mathcal{R}$, of degree equal to the rank of $G$.

(ii) If $G_{1}, \ldots, G_{n}$ are finite graded posets such that $G_{i} \not G_{j}$ for $1 \leq i<j \leq n$, then the elements $\overline{G_{1}}, \ldots, \overline{G_{n}}$ of $\mathcal{R}$ are linearly independent.

(iii) The multiplication of $\mathcal{R}$ is such that $\overline{G_{1}} \cdot \overline{G_{2}}=\overline{G_{1} \times G_{2}}$, where $G_{1} \times G_{2}$ denotes the direct product of the finite graded posets $G_{1}$ and $G_{2}$.

(iv) The comultiplication of $\mathcal{R}$ is such that for every finite graded poset $G$ one has

$$
\Delta(\bar{G})=\sum_{x \in G} \overline{\left[0_{G}, x\right]} \otimes \overline{\left[x, 1_{G}\right]},
$$

where we denote $\left[0_{G}, x\right]:=\{y \in G \mid y \leq x\}$ and $\left[x, 1_{G}\right]:=\{y \in G \mid y \geq x\}, x \in G$.

For a more detailed presentation of how $\mathcal{R}$ is constructed, see e.g. Section 3 of [12].

What makes $\mathcal{R}$ come into our considerations is that one has a natural embedding of $\mathcal{Y}^{(1)}$ into $\mathcal{R}$. More precisely, let $\Lambda: \mathcal{Y}^{(1)} \rightarrow \mathcal{R}$ be the unital algebra homomorphism uniquely determined by the requirement that

$$
\Lambda\left(Y_{n}\right)=\overline{N C(n)}, \quad \forall n \geq 2 .
$$

The assignment for $\Lambda\left(Y_{n}\right)$ in (7.17) makes sense, as $N C(n)$ is a finite graded poset of rank $n-1$ (see e.g. Exercise 10.29 on p. 171 of [11]). We have that $\Lambda$ is an injective homomorphism of graded Hopf algebras. The verification of this statement is left as an exercise to the reader, we only indicate references for the two details of the verification that are non-trivial.

(a) Why is $\Lambda$ injective: in view of (ii) and (iii) above, this comes down to the fact that if $2 \leq i_{1} \leq i_{2} \leq \cdots \leq i_{m}$ and $2 \leq j_{1} \leq j_{2} \leq \cdots \leq j_{n}$ are such that

$$
N C\left(i_{1}\right) \times \cdots \times N C\left(i_{m}\right) \simeq N C\left(j_{1}\right) \times \cdots \times N C\left(j_{n}\right),
$$

then $m=n$ and $i_{1}=j_{1}, \ldots, i_{n}=j_{n}$. For a proof of this fact, see Proposition 9.38 on p. 152 of [11].

(b) Why does $\Lambda$ respect comultiplication: in view of (iii) and (iv) above, this follows immediately from the fact that for $\pi=\left\{A_{1}, \ldots, A_{q}\right\} \in N C(n)$ with $K(\pi)=:\left\{B_{1}, \ldots, B_{r}\right\}$, the intervals $\left[0_{n}, \pi\right]$ and $\left[\pi, 1_{n}\right]$ of $N C(n)$ satisfy

$$
\begin{aligned}
& {\left[0_{n}, \pi\right] \simeq N C\left(\left|A_{1}\right|\right) \times \cdots \times N C\left(\left|A_{q}\right|\right),} \\
& {\left[\pi, 1_{n}\right] \simeq\left[0_{n}, K(\pi)\right] \simeq N C\left(\left|B_{1}\right|\right) \times \cdots \times N C\left(\left|B_{r}\right|\right) ;}
\end{aligned}
$$

see e.g. the discussion on pp. 149-150 of [11.

$2^{o}$ A benefit coming from the embedding of $\mathcal{Y}^{(1)}$ into $\mathcal{R}$ is that one gets a natural choice for a "zeta character" on $\mathcal{Y}^{(1)}$. Indeed, $\mathcal{R}$ has a zeta character $\zeta_{\mathcal{R}}$ which acts by

$$
\zeta_{\mathcal{R}}(\bar{G})=1, \quad \text { for every finite graded poset } G
$$


see Example 2.2 of [1]. By restricting to $\mathcal{Y}^{(1)}$ we thus see that the zeta character of $\mathcal{Y}^{(1)}$ should act by the prescription that

$$
\zeta_{\mathcal{Y}^{(1)}}\left(Y_{n}\right)=1, \quad \forall n \geq 2 .
$$

Now, a combinatorial Hopf algebra is defined in the paper [1] as a pair $(\mathcal{B}, \zeta)$ where $\mathcal{B}$ is a graded connected Hopf algebra and $\zeta$ is a character of $\mathcal{B}$. So in particular $\left(\mathcal{Y}^{(1)}, \zeta_{\mathcal{Y}^{(1)}}\right)$ is a combinatorial Hopf algebra, where $\mathcal{Y}^{(1)}$ is cocommutative. Theorem 4.3 of [1] asserts that if $(\mathcal{B}, \zeta)$ is a combinatorial Hopf algebra such that $\mathcal{B}$ is cocommutative, then there exists a unique homomorphism of graded Hopf algebras $\Psi: \mathcal{B} \rightarrow$ Sym such that $\zeta_{\text {sym }} \circ \Psi=\zeta$, where $\zeta_{\mathrm{Sym}} \in \mathbb{X}(\mathrm{Sym})$ is the special character determined by the requirement that

$$
\zeta_{\text {Sym }}\left(e_{1}\right)=1 \text { and } \zeta_{\text {Sym }}\left(e_{n}\right)=0 \text { for every } n \geq 2 .
$$

We claim that in the particular case when $(\mathcal{B}, \zeta)$ is $\left(\mathcal{Y}^{(1)}, \zeta_{\mathcal{Y}^{(1)}}\right)$, this gives us precisely the homomorphism $\Phi$ from Theorem 7.5. That is, we have the following proposition.

Proposition 7.8. $\zeta_{\text {Sym }} \circ \Phi=\zeta_{\mathcal{Y}^{(1)}}$.

Proof. For every $n \geq 2$ we have

$$
\begin{aligned}
& \left(\zeta_{\mathrm{Sym}} \circ \Phi\right)\left(Y_{n}\right)=\zeta_{\mathrm{Sym}}\left(y_{n}\right) \quad\left(\text { since } \Phi\left(Y_{n}\right)=y_{n}, \text { by Definition } 6.8,2\right) \\
= & \left.\sum_{\substack{\pi \in N C(n-1), \pi=\left\{A_{1}, \ldots, A_{q}\right\}}} \zeta_{\mathrm{Sym}}\left(e_{\left|A_{1}\right|}\right) \cdots \zeta_{\mathrm{Sym}}\left(e_{\left|A_{q}\right|}\right) \quad \text { (by def. of } y_{n} \text { in }(\underline{6.26})\right) .
\end{aligned}
$$

But from (7.19) it is clear that the only non-zero term in the sum (17.20) is the one corresponding to the partition $0_{n-1} \in N C(n-1)$, and this term is equal to 1 . So we obtain that $\left(\zeta_{\text {Sym }} \circ \Phi\right)\left(Y_{n}\right)=1=\zeta_{\mathcal{Y}^{(1)}}\left(Y_{n}\right), \forall n \geq 2$, and the proposition follows.

Remark 7.9. Having placed the homomorphism $\Phi$ in the framework of combinatorial Hopf algebras leads to an interesting alternative description of the symmetric functions $\left\{y_{n} \mid n \geq 2\right\}$, as linear combinations of monomial quasi-symmetric functions. For every $m$-tuple of positive integers $\left(r_{1}, \ldots, r_{m}\right)$, the corresponding monomial quasi-symmetric function $M_{\left(r_{1}, \ldots, r_{m}\right)}$ is defined as

$$
M_{\left(r_{1}, \ldots, r_{m}\right)}=\sum_{1 \leq i_{1}<i_{2}<\cdots<i_{m}} x_{i_{1}}^{r_{1}} x_{i_{2}}^{r_{2}} \cdots x_{i_{m}}^{r_{m}}
$$

where $\left\{x_{i} \mid i \geq 1\right\}$ is a family of commuting indeterminates. The elementary symmetric functions $e_{n}$ used earlier in the paper are of course a particular case of (7.21),

$$
e_{n}=M_{(}(\underbrace{1, \ldots, 1}_{n}), \quad \forall n \geq 1 .
$$

Hence one can take the point of view that Equation (6.26) defines $y_{n}$ as a sum of products of monomial quasi-symmetric functions, and one can expand these products in order to obtain 
$y_{n}$ as a linear combination of $M_{\left(r_{1}, \ldots, r_{m}\right)}$ 's with $r_{1}+\cdots+r_{m}=n-1$. To give a concrete example, for $n=4$ one has

$$
\begin{aligned}
y_{4} & =e_{3}+3 e_{1} e_{2}+e_{1}^{3} \quad(\text { as in }(\underline{6.27})) \\
& =M_{(1,1,1)}+3 M_{(1)} M_{(1,1)}+M_{(1)}^{3} \\
& =M_{(1,1,1)}+3\left(3 M_{(1,1,1)}+M_{(2,1)}+M_{(1,2)}\right)+\left(6 M_{(1,1,1)}+3 M_{(2,1)}+3 M_{(1,2)}+M_{(3)}\right) \\
& =16 M_{(1,1,1)}+6 M_{(2,1)}+6 M_{(1,2)}+M_{(3)} .
\end{aligned}
$$

In the above calculation for $n=4$ we ended up with a sum of 29 terms, which turn out to correspond to the $1+12+16$ chains in $N C(4)$ that were also used for illustration in Example 4.6. Indeed, the results about combinatorial Hopf algebras from Theorems 4.1 and 4.3 of [1] contain a concrete recipe for how to write $\Phi\left(Y_{n}\right)$ as a linear combination of monomial quasi-symmetric functions. Due to the embedding of $\mathcal{Y}^{(1)}$ into Rota's Hopf algebra from Remark 7.7,1 this recipe reduces to one previously found in Ehrenborg's paper [4] (see the discussion in Example 4.4 of [1]), and we get that

$$
y_{n}=\sum_{\substack{\Gamma=\left(\pi_{o}, \pi_{1}, \ldots, \pi_{\ell}\right) \\ \text { chain in } N C(n)}} M_{\left(\left|\pi_{0}\right|-\left|\pi_{1}\right|,\left|\pi_{1}\right|-\left|\pi_{2}\right|, \ldots,\left|\pi_{\ell-1}\right|-\left|\pi_{\ell}\right|\right), \quad \forall n \geq 2 .}
$$

It is likely that the equivalence between formulas (6.26) and (7.23) can be proved directly from the rules of how products of monomial quasi-symmetric functions are formed, but the argument for that doesn't seem to be immediate.

\section{Acknowledgements.}

The first-named author would like to thank Marcelo Aguiar and Frank Sottile for introducing him to combinatorial Hopf algebras.

The second-named author would like to thank Ed Effros, Sergey Fomin and Dan Voiculescu for inspiring discussions on the possible use of Hopf algebras in the combinatorics of free probability. Some of these discussions took place during two workshops at the American Institute of Mathematics in Palo Alto, in January 2005 and in June 2006; the second-named author gratefully acknowledges his participation in these workshops.

\section{References}

[1] M. Aguiar, N. Bergeron, F. Sottile. Combinatorial Hopf algebras and generalized DehnSomerville equations, Compositio Mathematica 142 (2006), 1-30.

[2] M. Anshelevich, E.G. Effros, M. Popa. Zimmermann type cancellation in the free Faa di Bruno algebra, Journal of Functional Analysis 237 (2006), 76-104.

[3] K. Dykema. Multilinear function series and transforms in free probability theory, Advances in Mathematics 208 (2007), 351-407.

[4] R. Ehrenborg. On posets and Hopf algebras, Advances in Mathematics 119 (1996), $1-25$. 
[5] H. Figueroa, J.M. Gracia-Bondia. Combinatorial Hopf algebras in quantum field theory I, Reviews in Mathematical Physics 17 (2005), 881-976.

[6] U. Haagerup. On Voiculescu's R- and S-transforms for free non-commuting random variables, in Free Probability Theory (D. Voiculescu, editor), Fields Institute Communications 12 (1997), 127-148.

[7] S.A. Joni, G.-C. Rota. Coalgebras and bialgebras in combinatorics, Studies in Applied Mathematics 61 (1979), 93-139.

[8] G. Kreweras. Sur les partitions non-croisées d'un cycle, Discrete Mathematics 1 (1972), 333-350.

[9] I.G. MacDonald. Symmetric functions and Hall polynomials, Clarendon Press, Oxford, 1979.

[10] A. Nica, R. Speicher. A "Fourier transform" for multiplicative functions on non-crossing partitions, Journal of Algebraic Combinatorics 6 (1997), 141-160.

[11] A. Nica, R. Speicher. Lectures on the combinatorics of free probability, London Mathematical Society Lecture Note Series 335, Cambridge University Press, 2006.

[12] W.R. Schmitt. Incidence Hopf algebras, Journal of Pure and Applied Algebra 96 (1994), 299-330.

[13] R. Simion. Noncrossing partitions, Discrete Mathematics 217 (2000), 367-409.

[14] R.P. Stanley. Enumerative combinatorics volume 2, Cambridge Studies in Advanced Mathematics 62, Cambridge University Press, 1999.

[15] M.E. Sweedler. Hopf algebras, Benjamin, 1969.

[16] D. Voiculescu. Multiplication of certain noncommuting random variables, Journal of Operator Theory 18 (1987), 223-235.

[17] D.V. Voiculescu, K.J. Dykema, A. Nica. Free random variables, CRM Monograph Series 1, American Mathematical Society, 1992.

[18] A.V. Zelevinsky. Representations of finite classical groups. A Hopf algebra approach, Lecture Notes in Mathematics 869, Springer Verlag, 1981.

Mitja Mastnak

Department of Pure Mathematics, University of Waterloo.

Current address: Department of Mathematics and Computer Science,

Saint Mary's University,

Halifax, Nova Scotia B3H 3C3, Canada.

Email: mmastnak@cs.smu.ca

Alexandru Nica

Department of Pure Mathematics, University of Waterloo,

Waterloo, Ontario N2L 3G1, Canada.

Email: anica@math.uwaterloo.ca 